\title{
Energy Saving Potential in Knuckle Boom Cranes using a Novel Pump Controlled Cylinder Drive
}

\author{
S. Ketelsen \\ L. Schmidt \\ V. H. Donkov \\ T. O. Andersen \\ Department of Energy Technology, Aalborg University, 9220 Aalborg, Denmark. E-mail: sok@et.aau.dk
}

\begin{abstract}
This paper is considering the application of a novel pump controlled cylinder drive, the so-called Speedvariable Switched Differential Pump (SvSDP), for knuckle boom crane actuation. Especially the control system for the SvSDP drive is considered, and aiming on improving energy efficiency a refinement of the existing control structure is proposed. An energy efficient sizing algorithm for the SvSDP drive is developed, and fundamental differences between the achievable operating range for the SvSDP drive compared to a conventional valve-cylinder drive are discussed. A case study is conducted with knuckle boom crane actuation, and compared to a conventional valve actuation. Simulation results show that the motion tracking performance is on a similar level compared to the valve actuation approach, while the energy consumption is drastically decreased. For the given test trajectory the valve actuation system consumes $0.79 \mathrm{kWh}$ of electrical energy, while the SvSDP drive consume $0.06 \mathrm{kWh}$, if ideal energy recovery and storage is assumed.
\end{abstract}

Keywords: Energy efficient hydraulic actuation, pump controlled cylinder, cylinder direct drive, offshore cranes, multivariable control

\section{Introduction}

The usage of hydraulics for low-speed high-force linear actuation is a well established standard in many industries. Hydraulic actuation are traditionally selected due to the high power and force density they can offer. Conventionally, hydraulic cylinders are controlled using proportional valves, which achieve the desired motion control performance by throttling the flows in and out of the cylinder chambers, which in turn is a major source of losses in hydraulic systems. To reduce the throttling losses, a load sensing pump may be installed. This is often the case in knuckle boom crane actuation systems, but a drawback of this strategy is that the supply pressure is determined by the demand of the consumer requiring the largest pressure. This may lead to situations where a fast moving unloaded cylinder, requires a large flow, while another small flow-high pressure consumer determines the sup- ply pressure, leading to large throttling losses for the fast moving cylinder. With increasing demands for energy efficieny in most industries, research communities as well as industry are trying to identify other methods for improving the overall efficiency of linear actuation in machinery, such as replacing hydraulic cylinders with self-contained electromechanical cylinders Hagen et al. (2017). Other researchers are looking into socalled digital hydraulics. Examples of this approach include multi-chamber and/or multi-pressure cylinders Hedegaard Hansen et al. (2018), Linjama et al. (2009) and Huova et al. (2017), hydraulic buck converters Kogler and Scheidl (2016), hydraulic power management concepts Vukovic et al. (2016), Linjama and Huhtala (2010) etc. Most of these technologies are still in the development phase, with the predominant challenges being low reliability under high load operating conditions for self-contained electromechanical cylinders Hagen et al. (2017), or the demand for faster 


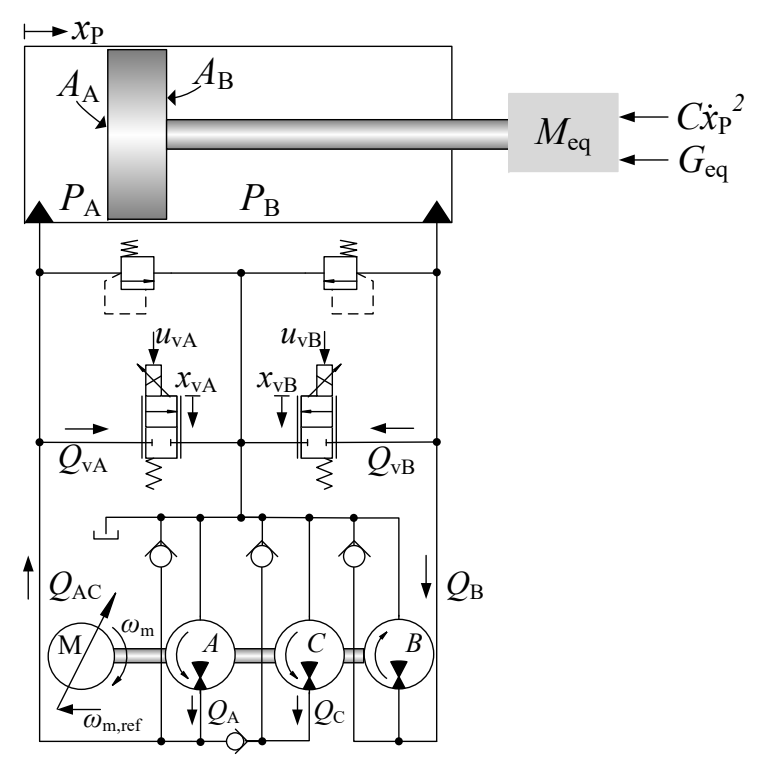

Figure 1: Asymmetric cylinder controlled by SvSDPsystem.

on/off valves for hydraulic buck converters Kogler and Scheidl (2016). Another approach for reducing throttling losses is direct hydraulic cylinder drives/pump controlled cylinders. The main idea is here to control the flow to the cylinder chamber directly by the pump without any valves in the main transmission lines. A fundamental challenge for this concept is that an asymmetric hydraulic cylinder requires different chamber volume flows. One way of compensating the unequal flow rates is by throttling only the differential volume flow using pilot operated check valves Rahmfeld (2002) or via an inverse shuttle valve Çaliskan et al. (2016) and Michel and Weber (2012). Another approach is to use an asymmetric pump unit Quan et al. (2014), which effectively also can be obtained by using two fixed displacement pumps such as investigated in Pedersen et al. (2014) and Järf et al. (2016). Here the two pumps are connected to a common shaft, but rotate in opposite directions. Furthermore, they are sized to match the area ratio of the cylinder.

The drive concept presented in Pedersen et al. (2014) demonstrated good hydraulic efficiency, but it was found that the effective pump displacement- and the cylinder area ratios, cannot be matched in the entire operating range due to pump leakage, resulting in irregular performance. These issues led to the development of the Speed-variable Switched Differential Pump (SvSDP) drive, which was introduced and investigated in Schmidt et al. (2015). The concept adds a third pump, only delivering flow in certain situations. In Schmidt et al. (2017) the control structure of this concept was further developed with the aim to decouple

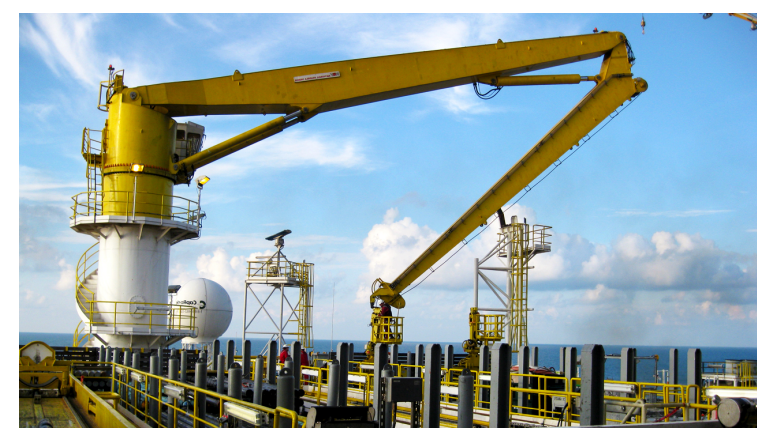

Figure 2: Knuckle Boom Crane example provided by National Oilwell Varco.(c)

the motion control and chamber pressure control.

The SvSDP drive topology is depicted in Fig. 1. At positive shaft speeds, pumps $\mathrm{A}$ and $\mathrm{C}$ provide flow to chamber A of the cylinder, while pump B withdraws fluid from cylinder chamber B. At negative shaft speeds pump C idles, effectively providing no flow to the cylinder A chamber. Hence a surplus flow into the cylinder is present in both directions of operation, and a pressure increase will appear for shaft speeds where pump flow exceeds internal leakage Schmidt et al. (2015). In order to maintain chamber pressures at reasonable levels this flow mismatch may be bled off via the $2 / 2$ proportional valves. Hence the system has three inputs $\omega_{\mathrm{m}, \mathrm{ref}}, u_{\mathrm{vA}}$ and $u_{\mathrm{vB}}$. As it is not possible to control the motion of the cylinder and both chamber pressures independently, only two sensible outputs may be defined, leaving the system over-actuated. In the current investigation, the SvSDP concept from Schmidt et al. (2017) will be investigated for actuation of a knuckle boom crane similar to the one seen in Fig. 2. A model based approach to the sizing of the SvSDP drives is presented, and a control strategy targeting at a high energy efficiency is established. The resulting performance is compared to that of a conventional valve operation approach and simulation results demonstrate similar motion performance, while the SvSDP drive approach shows a highly improved energy efficiency compared to the valve actuation system.

\section{Mechanical Knuckle Boom Crane Model}

A knuckle boom crane may be illustrated as depicted in Fig. 3. Due to the often large cylinders, the masses of cylinder tubes and rods may be significant compared to the crane booms and payload, and may therefore be taken into account. Hence the mechanical system may be depicted as having seven centers of mass (CMs), all moving relative to each other. As illustrated, the 
cylinder tube and piston CMs are equivalated into a combined CM for each cylinder, resulting in five CMs to be included in the model. Defining the joint angles $\varphi_{1}, \varphi_{2}$ and $\varphi_{3}$ (generalised coordinates), the CMs may be described by:

$$
\begin{aligned}
\mathbf{P}_{\mathrm{CM} 1} & =\mathbf{A}_{1} \mathbf{S}_{1}, \mathbf{P}_{\mathrm{CM} 2}=\mathbf{A}_{1} \mathbf{S}_{2}+\mathbf{A}_{2} \mathbf{S}_{3} \\
\mathbf{P}_{\mathrm{CM} 3} & =\mathbf{A}_{1} \mathbf{S}_{2}+\mathbf{A}_{2} \mathbf{S}_{4}+\mathbf{A}_{3} \mathbf{S}_{4}, \\
\mathbf{P}_{\mathrm{CMcyl} 1} & =\mathbf{A}_{4} \mathbf{S}_{6}, \mathbf{P}_{\mathrm{CMcyl} 2}=\mathbf{A}_{1} \mathbf{S}_{2}+\mathbf{A}_{5} \mathbf{S}_{7}
\end{aligned}
$$

Here, matrices and vectors are given by:

$$
\begin{aligned}
& \mathbf{A}_{1}=\left[\begin{array}{cc}
\cos \left(\varphi_{1}\right) & -\sin \left(\varphi_{1}\right) \\
\sin \left(\varphi_{1}\right) & \cos \left(\varphi_{1}\right)
\end{array}\right] \\
& \mathbf{A}_{2}=\left[\begin{array}{cc}
\cos \left(\varphi_{1}+\varphi_{2}\right) & -\sin \left(\varphi_{1}+\varphi_{2}\right) \\
\sin \left(\varphi_{1}+\varphi_{2}\right) & \cos \left(\varphi_{1}+\varphi_{2}\right)
\end{array}\right] \\
& \mathbf{A}_{3}=\left[\begin{array}{cc}
\cos \left(\varphi_{1}+\varphi_{2}+\varphi_{3}\right) & -\sin \left(\varphi_{1}+\varphi_{2}+\varphi_{3}\right) \\
\sin \left(\varphi_{1}+\varphi_{2}+\varphi_{3}\right) & \cos \left(\varphi_{1}+\varphi_{2}+\varphi_{3}\right)
\end{array}\right] \\
& \mathbf{A}_{4}=\left[\begin{array}{ll}
\cos \left(\varphi_{1}-\alpha_{8}-\alpha_{1}\right) & -\sin \left(\varphi_{1}-\alpha_{8}-\alpha_{1}\right) \\
\sin \left(\varphi_{1}-\alpha_{8}-\alpha_{1}\right) & \cos \left(\varphi_{1}-\alpha_{8}-\alpha_{1}\right)
\end{array}\right] \\
& \mathbf{A}_{5}=\left[\begin{array}{l}
\cos \left(\varphi_{1}+\varphi_{2}-\alpha_{10}+\alpha_{2}\right) \\
\sin \left(\varphi_{1}+\varphi_{2}-\alpha_{10}+\alpha_{2}\right) \\
\mathbf{S}_{1}=\left[\begin{array}{c}
\sin \left(\varphi_{1}+\varphi_{2}-\alpha_{10}+\alpha_{2}\right) \\
\cos \left(\varphi_{1}+\varphi_{2}-\alpha_{10}+\alpha_{2}\right)
\end{array}\right] \\
L_{\mathrm{CCM} 1 \mathrm{x}}
\end{array}\right], \mathbf{S}_{2}=\left[\begin{array}{c}
L_{\mathrm{CF}} \\
0
\end{array}\right], \mathbf{S}_{3}=\left[\begin{array}{c}
L_{\mathrm{FCM} 2 \mathrm{x}} \\
L_{\mathrm{FCM} 2 \mathrm{y}}
\end{array}\right]
\end{aligned}
$$

$$
\begin{aligned}
& \mathbf{S}_{4}=\left[\begin{array}{c}
L_{\mathrm{FJ}} \\
0
\end{array}\right], \mathbf{S}_{5}=\left[\begin{array}{c}
L_{\mathrm{JCM} 3} \\
0
\end{array}\right] \\
& \mathbf{S}_{6}=\left[\begin{array}{c}
L_{\mathrm{Ccyl} 1} \\
0
\end{array}\right], \mathbf{S}_{7}=\left[\begin{array}{c}
L_{\mathrm{Fcyl} 2} \\
0
\end{array}\right]
\end{aligned}
$$

The total kinetic energy $\mathcal{K}$ may be expressed as:

$$
\begin{aligned}
\mathcal{K} & =\frac{m_{1}}{2} \dot{\mathbf{P}}_{\mathrm{CM} 1}^{T} \dot{\mathbf{P}}_{\mathrm{CM} 1}+\frac{m_{2}}{2} \dot{\mathbf{P}}_{\mathrm{CM} 2}^{T} \dot{\mathbf{P}}_{\mathrm{CM} 2} \\
& +\frac{m_{3}}{2} \dot{\mathbf{P}}_{\mathrm{CM} 3}^{T} \dot{\mathbf{P}}_{\mathrm{CM} 3}+\frac{m_{\mathrm{cyl1} 1}}{2} \dot{\mathbf{P}}_{\mathrm{CMcyl} 1}^{T} \dot{\mathbf{P}}_{\mathrm{CMcyl} 1} \\
& +\frac{m_{\mathrm{cyl} 2}}{2} \dot{\mathbf{P}}_{\mathrm{CMcyl} 2}^{T} \dot{\mathbf{P}}_{\mathrm{CMcyl} 2}+\frac{J_{1}}{2} \dot{\varphi}_{1}^{2}+\frac{J_{2}}{2}\left(\dot{\varphi}_{1}+\dot{\varphi}_{2}\right)^{2} \\
& +\frac{J_{3}}{2}\left(\dot{\varphi}_{1}+\dot{\varphi}_{2}+\dot{\varphi}_{2}\right)^{2}+\frac{J_{\mathrm{cyl} 1}}{2}\left(\dot{\varphi}_{1}-\frac{\partial \alpha_{8}}{\partial \varphi_{1}} \dot{\varphi}_{1}\right) \\
& +\frac{J_{\mathrm{cyl} 2}}{2}\left(\dot{\varphi}_{1}+\dot{\varphi}_{2}-\frac{\partial \alpha_{10}}{\partial \varphi_{2}} \dot{\varphi}_{2}\right)
\end{aligned}
$$

The total potential energy $\mathcal{P}$ may be expressed as:

$$
\begin{aligned}
\mathcal{P} & =\mathbf{g}^{T}\left(\mathbf{P}_{\mathrm{CM} 1}+\mathbf{P}_{\mathrm{CM} 2}+\mathbf{P}_{\mathrm{CM} 3}+\mathbf{P}_{\mathrm{CMcyl} 1}\right. \\
& \left.+\mathbf{P}_{\mathrm{CMcyl} 2}\right), \quad \mathbf{g}=\left[\begin{array}{ll}
0 & g
\end{array}\right]^{T}
\end{aligned}
$$

Hence, the Lagrangian $\mathcal{L}=\mathcal{K}-\mathcal{P}$ may by formed, from which the joint torques may be established as:

$$
\begin{aligned}
& \tau_{1}=\frac{\mathrm{d}}{\mathrm{d} t} \frac{\partial \mathcal{L}}{\partial \dot{\varphi_{1}}}-\frac{\partial \mathcal{L}}{\partial \varphi_{1}} \\
& \tau_{2}=\frac{\mathrm{d}}{\mathrm{d} t} \frac{\partial \mathcal{L}}{\partial \dot{\varphi_{2}}}-\frac{\partial \mathcal{L}}{\partial \varphi_{2}} \\
& \tau_{3}=\frac{\mathrm{d}}{\mathrm{d} t} \frac{\partial \mathcal{L}}{\partial \dot{\varphi_{3}}}-\frac{\partial \mathcal{L}}{\partial \varphi_{3}}
\end{aligned}
$$

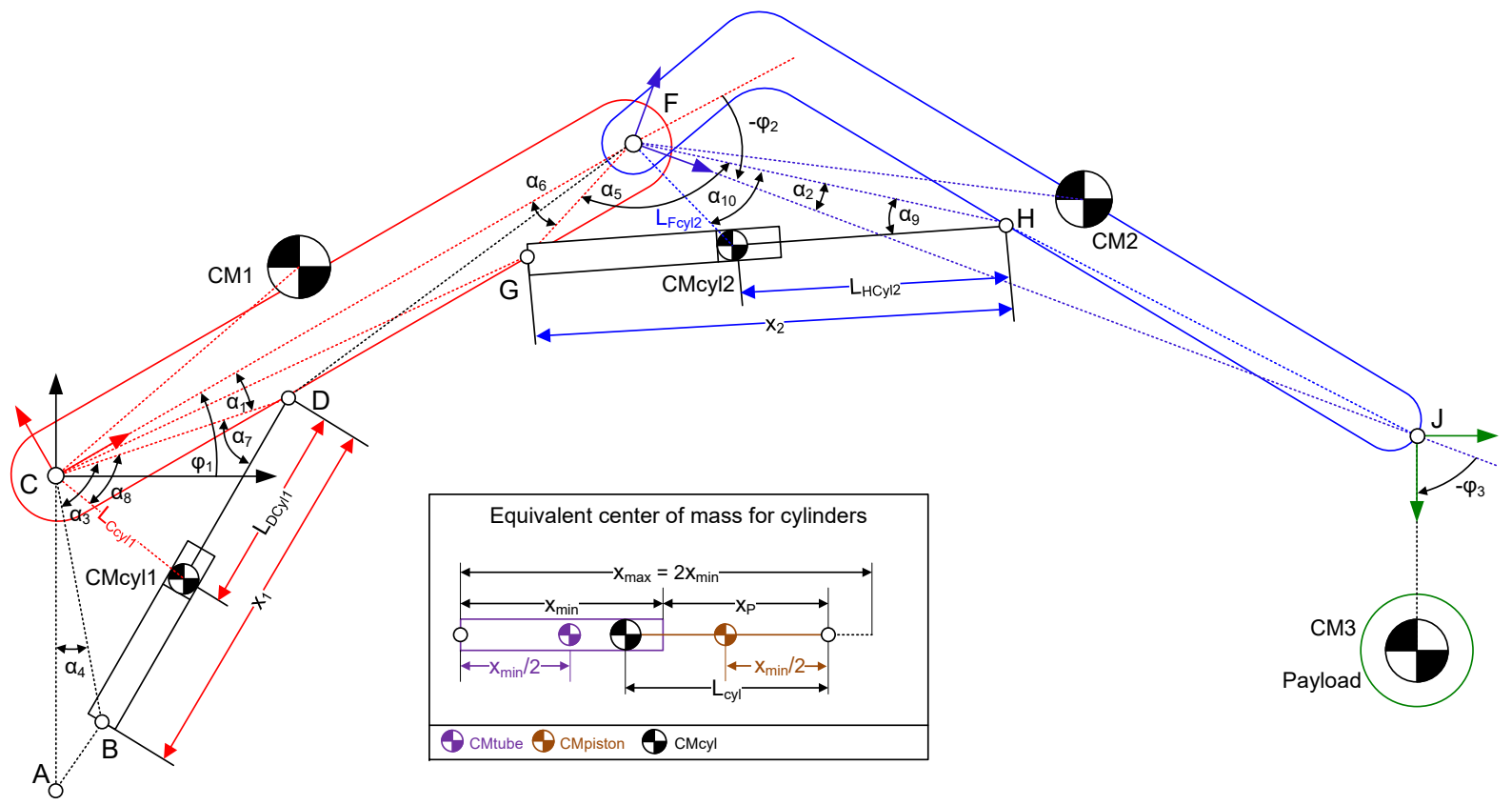

Figure 3: Illustration of a knuckle boom crane with two hydraulic cylinders. 
Defining $\mathbf{q}=\left[\begin{array}{lll}\varphi_{1} & \varphi_{2} & \varphi_{3}\end{array}\right]^{T}$ where $\varphi_{1}=\alpha_{1}+\alpha_{3}\left(x_{\mathrm{P} 1}\right)+$ $\alpha_{4}-\pi / 2, \varphi_{2}=\alpha_{5}\left(x_{\mathrm{P} 2}\right)+\alpha_{6}-\alpha_{2}-\pi$, the joint torque $\tau=\left[\begin{array}{lll}\tau_{1} & \tau_{2} & \tau_{3}\end{array}\right]^{T}$ may be expressed as:

$$
\tau=\mathbf{D}(\mathbf{q}) \ddot{\mathbf{q}}+\mathbf{C}(\mathbf{q}, \dot{\mathbf{q}})+\mathbf{G}(\mathbf{q})
$$

Noting $\dot{\mathbf{q}}=\mathbf{J} \dot{\mathbf{x}}, \dot{\mathbf{x}}=\left[\begin{array}{lll}\dot{x}_{\mathrm{P} 1} & \dot{x}_{\mathrm{P} 2} & 0\end{array}\right]^{T}$ the dynamic model may be described in cylinder space as:

$$
\begin{aligned}
\mathbf{F} & =\mathbf{J}^{T} \mathbf{D}(\mathbf{x}) \mathbf{J} \ddot{\mathbf{x}}+\mathbf{J}^{T}(\mathbf{D}(\mathbf{x}) \dot{\mathbf{J}} \dot{\mathbf{x}}+\mathbf{C}(\mathbf{x}, \dot{\mathbf{x}})) \\
& +\mathbf{J}^{T} \mathbf{G}(\mathbf{x})
\end{aligned}
$$

Here, $\mathbf{F}$ is the linear mechanical output force given by:

$$
\mathbf{F}=\mathbf{F}_{\mathrm{hyd}}-\mathbf{B} \dot{\mathbf{x}}, \quad \mathbf{B}=\left[\begin{array}{cc}
B_{\mathrm{v} 1} & 0 \\
0 & B_{\mathrm{v} 2}
\end{array}\right]
$$

\section{SvSDP Cylinder Drive}

The two structurally identical SvSDP drives needed for knuckle boom crane actuation are subscripted with $i=1,2\left(\operatorname{SvSDP}_{1}\right.$ and $\left.\operatorname{SvSDP}_{2}\right)$.

\subsection{Nonlinear Model}

Considering Fig. 1, the SvSDP drive is described by Eq. (12)-(20), assuming ideal check valves, no cylinder cross port leakage, nonlinear friction phenomena absent, and defining $V_{\mathrm{A}_{i}}=V_{\mathrm{A}_{i}}+A_{\mathrm{A}_{i}} x_{\mathrm{P}_{i}}, V_{\mathrm{B}_{i}}=$ $V_{\mathrm{B} 0_{i}}-A_{\mathrm{B}_{i}} x_{\mathrm{P}_{i}}, \alpha_{i}=A_{\mathrm{B}_{i}} / A_{\mathrm{A}_{i}} \cdot i=1,2$

$$
\begin{aligned}
& \ddot{x}_{\mathrm{P}_{i}}=\frac{A_{\mathrm{A}_{i}}\left(P_{\mathrm{A}_{i}}-\alpha_{i} P_{\mathrm{B}_{i}}\right)-\dot{x}_{\mathrm{P}_{i}} B_{\mathrm{v}_{i}}-f(x, \dot{x})}{M_{\mathrm{eq}_{i}}} \\
& \dot{P}_{\mathrm{A}_{i}}=\frac{\beta_{\mathrm{A}_{i}}}{V_{\mathrm{A}_{i}}}\left(Q_{\mathrm{AC}_{i}}-Q_{\mathrm{vA}_{i}}-\dot{x}_{\mathrm{P}_{i}} A_{\mathrm{A}_{i}}\right) \\
& \dot{P}_{\mathrm{B}_{i}}=\frac{\beta_{\mathrm{B}_{i}}}{V_{\mathrm{B}_{i}}}\left(\dot{x}_{\mathrm{P}_{i}} \alpha_{i} A_{\mathrm{A}_{i}}-Q_{\mathrm{B}_{i}}-Q_{\mathrm{vB}_{i}}\right) \\
& Q_{\mathrm{AC}_{i}}= \begin{cases}\bar{Q}_{\mathrm{A}_{i}}\left(\omega_{\mathrm{m}_{i}}\right) \eta_{\mathrm{vA}_{i}}+\bar{Q}_{\mathrm{C}_{i}}\left(\omega_{\mathrm{m}_{i}}\right) \eta_{\mathrm{vC}_{i}}, & , \omega_{\mathrm{m}_{i}} \geq 0 \\
\bar{Q}_{\mathrm{A}_{i}}\left(\omega_{\mathrm{m}_{i}}\right) / \eta_{\mathrm{vA}_{i}} & , \omega_{\mathrm{m}_{i}}<0\end{cases} \\
& Q_{\mathrm{B}_{i}}=\left\{\begin{array}{lll}
\bar{Q}_{\mathrm{B}_{i}}\left(\omega_{\mathrm{m}_{i}}\right) / \eta_{\mathrm{vB}_{i}} & , \quad \omega_{\mathrm{m}_{i}} \geq 0 \\
\bar{Q}_{\mathrm{B}_{i}}\left(\omega_{\mathrm{m}_{i}}\right) \eta_{\mathrm{vB}} & , \quad \omega_{\mathrm{m}_{i}}<0
\end{array}\right. \\
& Q_{\mathrm{vA}_{i}}=K_{\mathrm{vA}_{i}} x_{\mathrm{vA}_{i}}, \quad Q_{\mathrm{vB}_{i}}=K_{\mathrm{vB}_{i}} x_{\mathrm{vB}_{i}} \\
& \ddot{x}_{\mathrm{vA}_{i}}=\omega_{\mathrm{vA}_{i}}^{2} u_{\mathrm{vA}_{i}}-2 \zeta_{\mathrm{vA}_{i}} \omega_{\mathrm{vA}_{i}} \dot{x}_{\mathrm{vA}_{i}}-\omega_{\mathrm{vA}}^{2} x_{\mathrm{vA}_{i}} \\
& \ddot{x}_{\mathrm{vB}_{i}}=\omega_{\mathrm{vB}_{i}}^{2} u_{\mathrm{vB}_{i}}-2 \zeta_{\mathrm{vB}_{i}} \omega_{\mathrm{vB}_{i}} \dot{x}_{\mathrm{vB}_{i}}-\omega_{\mathrm{vB}}^{2} x_{\mathrm{vB}_{i}} \\
& \ddot{\omega}_{\mathrm{m}_{i}}=\omega_{\mathrm{v} i}^{2} \omega_{\mathrm{m}, \mathrm{ref}_{i}}-2 \zeta_{\mathrm{v}_{i}} \omega_{\mathrm{v}_{i}} \dot{\omega}_{\mathrm{m}_{i}}-\omega_{\mathrm{v}}^{2} \omega_{\mathrm{m}_{i}}
\end{aligned}
$$

$f(x, \dot{x})$ contains the gravitational load and the Coriolis force. $\bar{Q}_{\mathrm{A}_{i}}, \bar{Q}_{\mathrm{B}_{i}}$ and $\bar{Q}_{\mathrm{C}_{i}}$ are leakage free pump flows, $\eta_{\mathrm{vA}_{i}}, \eta_{\mathrm{vB}_{i}}$ and $\eta_{\mathrm{vC}_{i}}$ are pressure dependent volumetric pump efficiencies, $P_{\mathrm{A}_{i}}$ and $P_{\mathrm{B}_{i}}$ chamber pressures, $x_{\mathrm{vA}}, x_{\mathrm{vB}_{i}}$ the valve spool positions, $\omega_{\mathrm{m}_{i}}$ the motor shaft speed and $x_{\mathrm{P}_{i}}$ the cylinder piston position. The valve inputs $u_{\mathrm{vA}_{i}}, u_{\mathrm{vB}_{i}}$ and the motor shaft reference speed $\omega_{\mathrm{m}, \mathrm{ref}_{i}}$ are the three system inputs. Additionally, $B_{\mathrm{v}, i}$ is a viscous friction coefficient, $\zeta_{\mathrm{vA}_{i}}, \zeta_{\mathrm{vB}_{i}}, \zeta_{\mathrm{v}_{i}}$ damping ratios, $\omega_{\mathrm{vA}_{i}}, \omega_{\mathrm{vB}_{i}}, \omega_{\mathrm{v}_{i}}$ bandwidths, $\beta_{\mathrm{A}_{i}}, \beta_{\mathrm{B}_{i}}$ the effective bulk moduli and $K_{\mathrm{vA}}, K_{\mathrm{vB}}$ are the flow gains of the $2 / 2$ pressure compensated proportional valves.

Furthermore, the hydraulic forces are given by:

$$
\mathbf{F}_{\text {hyd }}=\left[\begin{array}{c}
A_{\mathrm{A}_{1}}\left(P_{\mathrm{A}_{1}}-\alpha_{1} P_{\mathrm{B}_{1}}\right) \\
A_{\mathrm{A}_{2}}\left(P_{\mathrm{A}_{2}}-\alpha_{2} P_{\mathrm{B}_{2}}\right)
\end{array}\right]
$$

\subsection{Linear Model}

The inverse flow characteristics of the $2 / 2$ proportional valves are used to compensate the input $\left(u_{\mathrm{vA}_{i}}\right.$ and $\left.u_{\mathrm{vB}_{i}}\right)$, meaning that ideally $Q_{\mathrm{vA}_{i}}=Q_{\mathrm{vA}_{\text {, }} \text { fef }_{i}}, Q_{\mathrm{vB}_{i}}=$ $Q_{\mathrm{vB}, \text { ref }_{i}}$, if neglecting valve dynamics. Considering the gravitational load as a disturbance and assuming the velocity dependent Coriolis forces negligible and equal effective bulk moduli (i.e. $\beta_{\mathrm{A}_{i}}=\beta_{\mathrm{B}_{i}}=\beta_{i}$ ), the linear model is given by Eq. (22), when defining relations $\rho_{i}=V_{\mathrm{B}_{i}} / V_{\mathrm{A}_{i}}, \rho_{0_{i}}=V_{0 \mathrm{~B}_{i}} / V_{0 \mathrm{~A}_{i}}$ where $V_{0 \mathrm{~A}_{i}}=\left.V_{\mathrm{A}_{i}}\right|_{\mathbf{x}_{0_{i}}}$, $V_{0 \mathrm{~B}_{i}}=\left.V_{\mathrm{B}_{i}}\right|_{\mathbf{x}_{0_{i}}}$ and $\mathbf{x}_{0_{i}}$ is the state vector at the linearisation point.

$$
\begin{aligned}
& \dot{\mathbf{x}}_{\mathrm{c}}=\mathbf{A}_{\mathrm{c}_{i}} \mathbf{x}_{\mathrm{c}_{i}}+\mathbf{B}_{\mathrm{c}_{i}} \mathbf{u}_{\mathrm{ref}_{i}}, \quad \mathbf{y}_{\mathrm{p}_{i}}=\mathbf{C}_{\mathrm{c}_{i}} \mathbf{x}_{\mathrm{c}_{i}} \\
& \mathbf{x}_{\mathrm{c}_{i}}=\left[\begin{array}{c}
\mathbf{x}_{\mathrm{p}_{i}} \\
\mathbf{x}_{\mathrm{u}_{i}}
\end{array}\right], \mathbf{A}_{\mathrm{c}_{i}}=\left[\begin{array}{cc}
\mathbf{A}_{\mathrm{p}_{i}} & \mathbf{B}_{\mathrm{p}_{i}} \mathbf{C}_{\mathrm{u}_{i}} \\
\mathbf{0} & \mathbf{A}_{\mathrm{u}_{i}}
\end{array}\right] \\
& \mathbf{B}_{\mathrm{c}_{i}}=\left[\begin{array}{c}
\mathbf{0} \\
\mathbf{B}_{\mathrm{u}_{i}}
\end{array}\right], \mathbf{C}_{\mathrm{c}_{i}}=\left[\begin{array}{ll}
\mathbf{C}_{\mathrm{p}_{i}} & \mathbf{0}
\end{array}\right] \\
& \mathbf{x}_{\mathrm{p}_{i}}=\left[\begin{array}{llll}
x_{\mathrm{P}_{i}} & \dot{x}_{\mathrm{P}_{i}} & p_{\mathrm{A}_{i}} & p_{\mathrm{B}_{i}}
\end{array}\right]^{T} \\
& \mathbf{x}_{\mathrm{u}_{i}}=\left[\begin{array}{llllll}
\omega_{\mathrm{m}_{i}} & \dot{\omega}_{\mathrm{m}_{i}} & q_{\mathrm{vA}_{i}} & \dot{q}_{\mathrm{vA}_{i}} & q_{\mathrm{vB}_{i}} & \dot{q}_{\mathrm{vB}_{i}}
\end{array}\right]^{T} \\
& \mathbf{u}_{\text {ref }_{i}}=\left[\begin{array}{lll}
\omega_{\mathrm{m}_{\text {,ref }}} & q_{\mathrm{vA}, \mathrm{ref}_{i}} & q_{\mathrm{vB}, \mathrm{ref}_{i}}
\end{array}\right]^{T} \\
& \mathbf{y}_{\mathrm{p}_{i}}=\left[\begin{array}{lll}
x_{\mathrm{P}_{i}} & p_{\mathrm{A}_{i}} & p_{\mathrm{B}_{i}}
\end{array}\right]^{T}, \mathbf{C}_{\mathrm{p}_{i}}=\left[\begin{array}{cccc}
1 & 0 & 0 & 0 \\
0 & 0 & 1 & 0 \\
0 & 0 & 0 & 1
\end{array}\right]
\end{aligned}
$$

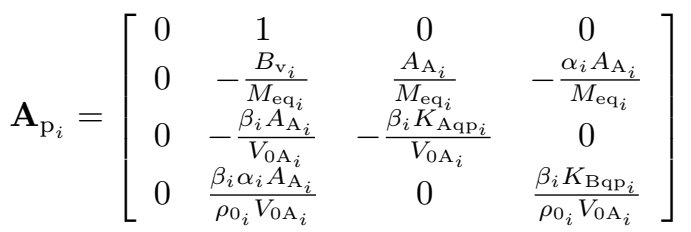

$$
\begin{aligned}
& \mathbf{B}_{\mathrm{p}_{i}}=\left[\begin{array}{ccc}
0 & 0 & 0 \\
0 & 0 & 0 \\
\frac{\beta_{i} K_{\mathrm{Aq}_{i}}}{V_{0 \mathrm{~A}_{i}}} & -\frac{\beta_{i}}{V_{0 \mathrm{~A}_{i}}} & 0 \\
-\frac{\beta_{i} K_{\mathrm{Bq}_{i}}}{\rho_{0_{i}} V_{0 \mathrm{~A}_{i}}} & 0 & -\frac{\beta_{i}}{\rho_{0_{i}} V_{0 \mathrm{~A}_{i}}}
\end{array}\right] \\
& K_{\mathrm{Aqp}_{i}}=\left.\frac{\partial Q_{\mathrm{AC}_{i}}}{\partial P_{\mathrm{A}_{i}}}\right|_{\mathbf{x}_{0}} \quad K_{\mathrm{Bqp}_{i}}=\left.\frac{\partial Q_{\mathrm{B}_{i}}}{\partial P_{\mathrm{B}_{i}}}\right|_{\mathbf{x}_{0}} \\
& K_{\mathrm{Aq}_{i}}=\left.\frac{\partial Q_{\mathrm{AC}_{i}}}{\partial \omega_{\mathrm{m}_{i}}}\right|_{\mathbf{x}_{0}} \quad K_{\mathrm{Bq}_{i}}=\left.\frac{\partial Q_{\mathrm{B}_{i}}}{\partial \omega_{\mathrm{m}_{i}}}\right|_{\mathbf{x}_{0}}
\end{aligned}
$$




$$
\begin{aligned}
& \mathbf{A}_{\mathbf{u}_{i}}=\left[\begin{array}{ccc}
\mathbf{A}_{\mathrm{u}_{i}} & \mathbf{0} & \mathbf{0} \\
\mathbf{0} & \mathbf{A}_{\mathrm{u}_{2}} & \mathbf{0} \\
\mathbf{0} & \mathbf{0} & \mathbf{A}_{\mathbf{u} 3_{i}}
\end{array}\right] \\
& \mathbf{A}_{\mathrm{u} 1_{i}}=\left[\begin{array}{cc}
0 & 1 \\
-\omega_{\mathrm{v}_{i}}^{2} & -2 \zeta_{\mathrm{v}_{i}} \omega_{\mathrm{v}_{i}}
\end{array}\right] \\
& \mathbf{A}_{\mathrm{u} 2_{i}}=\left[\begin{array}{cc}
0 & 1 \\
-\omega_{\mathrm{vA}_{i}}^{2} & -2 \zeta_{\mathrm{vA}_{i}} \omega_{\mathrm{vA}_{i}}
\end{array}\right] \\
& \mathbf{C}_{\mathrm{u}_{i}}=\left[\begin{array}{cccccc}
1 & 0 & 0 & 0 & 0 & 0 \\
0 & 0 & 1 & 0 & 0 & 0 \\
0 & 0 & 0 & 0 & 1 & 0
\end{array}\right] \\
& \mathbf{B}_{\mathrm{u}_{i}}=\left[\begin{array}{ccc}
0 & 0 & 0 \\
\omega_{\mathrm{v}_{i}}^{2} & 0 & 0 \\
0 & 0 & 0 \\
0 & \omega_{\mathrm{vA}_{i}}^{2} & 0 \\
0 & 0 & 0 \\
0 & 0 & \omega_{\mathrm{vB}_{i}}^{2}
\end{array}\right]
\end{aligned}
$$

The combined linear model Eq. (22) may be expressed by the transfer function matrix Eq. (24)

$$
\begin{aligned}
& \mathbf{y}_{\mathrm{p}}(s)=\mathbf{G}_{\mathrm{c}}(s) \mathbf{u}_{\mathrm{ref}}(s) \\
& \mathbf{G}_{\mathrm{c}}(s)=\mathbf{C}_{\mathrm{c}}\left(s \mathbf{I}-\mathbf{A}_{\mathrm{c}}\right)^{-1} \mathbf{B}_{\mathrm{c}}
\end{aligned}
$$

The transfer functions for the plant and actuator dynamics may respectively be obtained as:

$$
\begin{aligned}
& \mathbf{y}_{\mathbf{p}_{i}}(s)=\mathbf{G}_{\mathbf{p}_{i}}(s) \mathbf{u}_{\mathrm{p}_{i}}(s) \\
& \mathbf{G}_{\mathrm{p}_{i}}(s)=\mathbf{C}_{\mathbf{p}_{i}}\left(s \mathbf{I}-\mathbf{A}_{\mathbf{p}_{i}}\right)^{-1} \mathbf{B}_{\mathbf{p}_{i}} \\
& \mathbf{y}_{\mathbf{u}_{i}}(s)=\mathbf{G}_{\mathbf{u}_{i}}(s) \mathbf{u}_{\mathrm{ref}_{i}}(s) \\
& \mathbf{G}_{\mathrm{u}_{i}}(s)=\mathbf{C}_{\mathbf{u}_{i}}\left(s \mathbf{I}-\mathbf{A}_{\mathbf{u}_{i}}\right)^{-1} \mathbf{B}_{\mathbf{u}_{i}} \\
& \mathbf{u}_{\mathrm{p}_{i}}=\left[\begin{array}{lll}
\omega_{\mathrm{m}_{i}} & q_{\mathrm{vA}_{i}} q_{\mathrm{vB}_{i}}
\end{array}\right]^{T} \\
& \mathbf{u}_{\mathrm{ref}_{i}}=\left[\begin{array}{lll}
\omega_{\mathrm{m}, \mathrm{ref}_{i}} & q_{\mathrm{vA}, \mathrm{ref}_{i}} q_{\mathrm{vB}, \mathrm{ref}_{i}}
\end{array}\right]^{T}
\end{aligned}
$$

In Schmidt et al. (2017) the significance of the dynamic couplings was studied using a relative gain array (RGA)-analysis. For the considered input-output combinations it was found that severe dynamic couplings are present, especially close to the system eigenfrequency. Due to these couplings a decentralised control strategy may not be utilised directly on the system.

\subsection{Control Strategy}

In Schmidt et al. (2017) a drive control strategy has been developed to handle the dynamic couplings, with the overall structure depicted in Fig. 4. The fundamental idea is to transform the input- and output variables using $\tilde{\mathbf{y}}=\mathbf{W}_{2} \mathbf{y}_{\mathrm{p}}, \tilde{\mathbf{u}}=\mathbf{W}_{1}^{-1} \mathbf{u}_{\text {ref. }}$. By choosing the transformation matrices $\mathbf{W}_{1}$ and $\mathbf{W}_{2}$ properly it is shown possible to decouple the transformed system states, as shown in Eq. (27) and Eq. (28).

$$
\begin{array}{r}
\mathbf{u}_{\mathrm{p}}=\mathbf{G}_{\mathrm{u}} \mathbf{u}_{\mathrm{ref}}, \mathbf{u}_{\mathrm{ref}}=\mathbf{W}_{1} \tilde{\mathbf{u}} \Rightarrow \mathbf{u}_{\mathrm{p}}=\mathbf{G}_{\mathrm{u}} \mathbf{W}_{1} \tilde{\mathbf{u}} \\
\tilde{\mathbf{y}}=\mathbf{W}_{2} \mathbf{y}_{\mathrm{p}}, \mathbf{y}_{\mathrm{p}}=\mathbf{G}_{\mathrm{p}} \mathbf{u}_{\mathrm{p}} \Rightarrow \tilde{\mathbf{y}}=\mathbf{W}_{2} \mathbf{G}_{\mathrm{p}} \mathbf{u}_{\mathrm{p}}
\end{array}
$$

Substituting Eq. (27) into Eq. (28), gives the transformed system as:

$$
\tilde{\mathbf{y}}=\mathbf{W}_{2} \mathbf{G}_{\mathrm{p}} \mathbf{G}_{\mathbf{u}} \mathbf{W}_{1} \tilde{\mathbf{u}}=\tilde{\mathbf{G}}_{\mathrm{c}} \tilde{\mathbf{u}}
$$

Note that the index $i$ is omitted in this section, as the developed control strategy is identical for both cylinder drives.

\subsubsection{Output Transformation}

As mentioned, it is only sensible to control two of the non-transformed outputs. As three inputs are available the system is said to be over-actuated. In Schmidt et al. (2017) it is found desirable to formulate an output transformation $\left(\mathbf{W}_{2}\right)$ such that more appropriate states than the actual chamber pressures may be considered. These appropriate states are selected to be the piston position, the virtual load pressure $P_{\mathrm{L}}$, and the level pressure $P_{\delta}$. The level pressure can be considered a weighted sum of the chamber pressures:

$$
P_{\mathrm{L}}=P_{\mathrm{A}}-\alpha P_{\mathrm{B}}, \quad P_{\delta}=P_{\mathrm{A}}+\delta P_{\mathrm{B}}, \quad \delta>0
$$

Using $P_{\mathrm{L}}, P_{\delta}$ then $P_{\mathrm{A}}, P_{\mathrm{B}}$ may be written as:

$$
P_{\mathrm{A}}=\frac{\alpha P_{\delta}}{\alpha+\delta}+\frac{\delta P_{\mathrm{L}}}{\alpha+\delta}, P_{\mathrm{B}}=\frac{P_{\delta}}{\alpha+\delta}-\frac{P_{\mathrm{L}}}{\alpha+\delta}
$$

The nonlinear dynamics of the load and level pressure are described in Eq. (32) and Eq. (33).

$$
\begin{aligned}
\dot{P}_{\mathrm{L}} & =\dot{P}_{\mathrm{A}}-\alpha \dot{P}_{\mathrm{B}} \\
& =\frac{\beta}{\rho V_{\mathrm{A}}}\left(\rho\left(Q_{\mathrm{AC}}-Q_{\mathrm{vA}}\right)+\alpha\left(Q_{\mathrm{B}}+Q_{\mathrm{vB}}\right)\right. \\
& \left.-A_{\mathrm{A}}\left(\alpha^{2}+\rho\right) \dot{x}_{\mathrm{P}}\right) \\
\dot{P}_{\delta} & =\dot{P}_{\mathrm{A}}+\delta \dot{P}_{\mathrm{B}}+\dot{\delta} P_{\mathrm{B}} \\
& =\frac{\beta}{\rho V_{\mathrm{A}}}\left(\rho\left(Q_{\mathrm{AC}}-Q_{\mathrm{vA}}\right)-\delta\left(Q_{\mathrm{B}}+Q_{\mathrm{vB}}\right)\right. \\
& \left.-A_{\mathrm{A}}(\rho-\alpha \delta) \dot{x}_{\mathrm{P}}\right)+\frac{\dot{\delta}}{\delta+\alpha}\left(P_{\delta}-P_{\mathrm{L}}\right)
\end{aligned}
$$

In Eq. (33) $\delta$ is chosen as $\rho / \alpha$ in order to decouple volume flow from the level pressure dynamics. Doing so, Eq. (32) and Eq. (33) become:

$$
\begin{aligned}
\dot{P}_{\mathrm{L}} & =\frac{\beta}{\rho V_{\mathrm{A}}}\left(\rho\left(Q_{\mathrm{AC}}-Q_{\mathrm{vA}}\right)+\alpha\left(Q_{\mathrm{B}}+Q_{\mathrm{vB}}\right)\right. \\
& \left.-A_{\mathrm{A}}\left(\alpha^{2}+\rho\right) \dot{x}_{\mathrm{P}}\right) \\
\dot{P}_{\delta} & =\frac{\beta}{\rho V_{\mathrm{A}}}\left(\rho\left(Q_{\mathrm{AC}}-Q_{\mathrm{vA}}\right)-\delta\left(Q_{\mathrm{B}}+Q_{\mathrm{vB}}\right)\right. \\
& \left.-\dot{x}_{\mathrm{P}} \frac{A_{\mathrm{A}} \rho}{\beta} \frac{\delta+1}{\delta+\alpha}\left(P_{\delta}-P_{\mathrm{L}}\right)\right)
\end{aligned}
$$




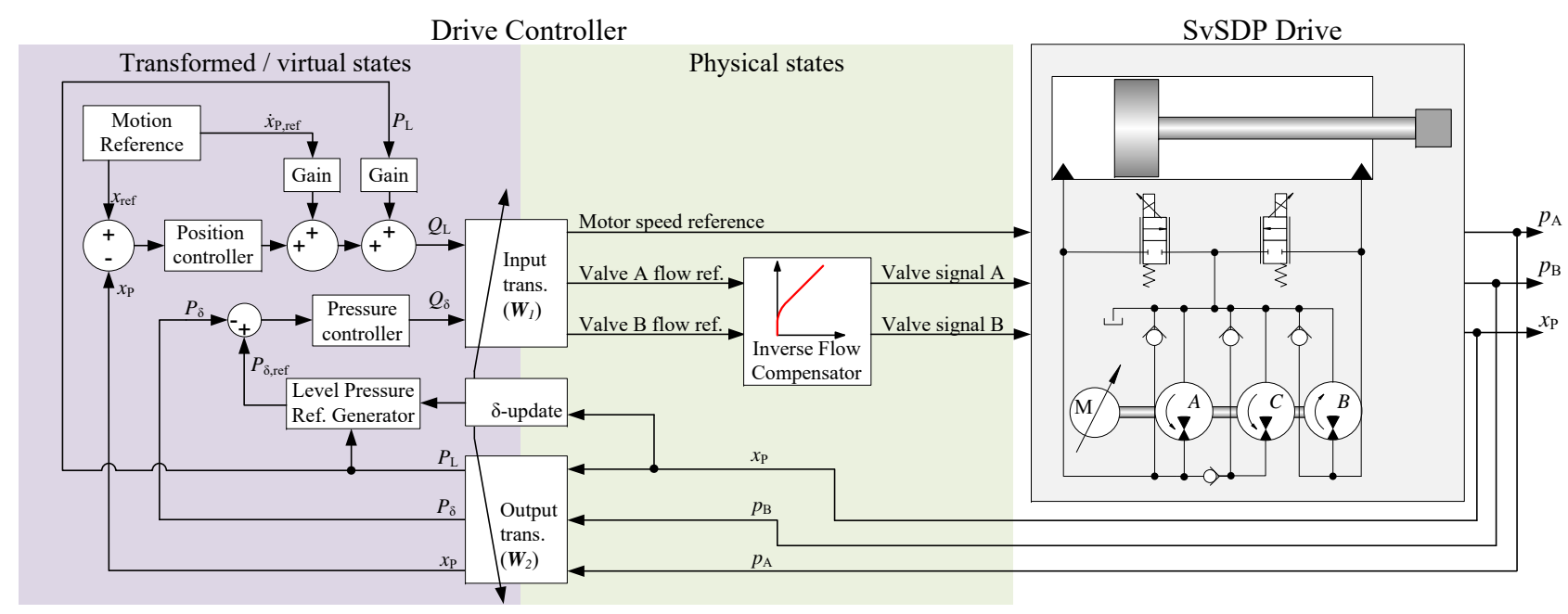

Figure 4: Schematic of the complete drive control system. Schmidt et al. (2017).

The linear pressure dynamics may be obtained as:

$$
\begin{aligned}
\dot{p}_{\delta_{0}} & =\frac{\beta}{V_{0 \mathrm{~A}}\left(\alpha+\delta_{0}\right)}\left(\left(\alpha+\delta_{0}\right)\left(K_{\Delta \omega} \omega_{\mathrm{m}}-q_{\mathrm{vA}}-\frac{q_{\mathrm{vB}}}{\alpha}\right)\right. \\
& \left.-\left(K_{\delta \mathrm{p} \delta}+K_{\delta \mathrm{p}}\right) p_{\delta}-\left(K_{\delta \mathrm{pL}}-K_{\delta \mathrm{p}}\right) p_{\mathrm{L}}\right) \\
& -K_{\delta \mathrm{xd}} \dot{x}_{\mathrm{P}}-K_{\delta \mathrm{xp}} x_{\mathrm{P}} \\
\dot{p}_{\mathrm{L}} & =\frac{\beta\left(\alpha+\delta_{0}\right)}{V_{0 \mathrm{~A}} \delta_{0}}\left(\frac{\delta_{0}}{\alpha+\delta_{0}}\left(K_{\Lambda \omega} \omega_{\mathrm{m}}-q_{\mathrm{vA}}+\frac{q_{\mathrm{vB}}}{\delta_{0}}\right)\right. \\
& \left.-A_{\mathrm{A}} \dot{x}_{\mathrm{P}}-\frac{\delta_{0} K_{\mathrm{Lp} \delta}}{\left(\alpha+\delta_{0}\right)^{2}} p_{\delta}-\frac{\delta_{0} K_{\mathrm{LpL}}}{\left(\alpha+\delta_{0}\right)^{2}} p_{\mathrm{L}}\right) \\
K_{\Delta \omega} & =K_{\mathrm{Aq}}-\frac{K_{\mathrm{Bq}}}{\alpha}, K_{\delta \mathrm{p} \delta}=\alpha K_{\mathrm{Aqp}}-\frac{K_{\mathrm{Bqp}}}{\alpha} \\
K_{\delta \mathrm{pL}} & =\delta_{0} K_{\mathrm{Aqp}}+\frac{K_{\mathrm{Bqp}}}{\alpha}, K_{\Lambda \omega}=K_{\mathrm{Aq}}+\frac{K_{\mathrm{Bq}}}{\delta_{0}} \\
K_{\mathrm{Lp} \delta}= & \alpha K_{\mathrm{Aqp}}+\frac{K_{B q p}}{\delta_{0}}, K_{\mathrm{LpL}}=\delta_{0} K_{\mathrm{Aqp}}-\frac{K_{\mathrm{Bqp}}}{\delta_{0}} \\
K_{\delta \mathrm{p}}= & \frac{\dot{x}_{\mathrm{P} 0} A_{\mathrm{A}}\left(\delta_{0}+1\right)}{\beta}, K_{\delta \mathrm{xd}}=\left.\frac{\partial \dot{P}_{\delta}}{\partial \dot{x}_{P}}\right|_{\mathbf{x}_{0}} \\
K_{\delta \mathrm{xp}}= & \left.\frac{\partial \dot{P}_{\delta}}{\partial x_{\mathrm{P}}}\right|_{\mathbf{x}_{0}}
\end{aligned}
$$

From the above, the output transformation may be established as:

$$
\tilde{\mathbf{y}}=\mathbf{W}_{2} \mathbf{y}, \tilde{\mathbf{y}}=\left[\begin{array}{c}
x_{\mathrm{P}} \\
p_{\mathrm{L}} \\
p_{\delta}
\end{array}\right], \mathbf{W}_{2}=\left[\begin{array}{ccc}
1 & 0 & 0 \\
0 & 1 & -\alpha \\
0 & 1 & \delta_{0}
\end{array}\right]
$$

Virtual inputs in terms of level flow $q_{\delta}$ and load flow $q_{\mathrm{L}}$ are defined based on the level and load pressure dynamics in Eq. (36) and Eq. (37), as defined in Eq.
(39) and Eq. (40):

$$
\begin{aligned}
q_{\delta} & =\left(\alpha+\delta_{0}\right)\left(K_{\Delta \omega} \omega_{\mathrm{m}}-q_{\mathrm{vA}}-\frac{q_{\mathrm{vB}}}{\alpha}\right) \\
q_{\mathrm{L}} & =\frac{\delta_{0}}{\alpha+\delta_{0}}\left(K_{\Lambda \omega} \omega_{m}-q_{\mathrm{vA}}+\frac{q_{\mathrm{vB}}}{\delta_{0}}\right) \\
\dot{p}_{\delta_{0}} & =\frac{\beta}{V_{0 \mathrm{~A}}\left(\alpha+\delta_{0}\right)}\left(q_{\delta}-\left(K_{\delta \mathrm{p} \delta}+K_{\delta \mathrm{p}}\right) p_{\delta}-\right. \\
& \left.\left(K_{\delta \mathrm{pL}}-K_{\delta \mathrm{p}}\right) p_{\mathrm{L}}\right)-K_{\delta \mathrm{xd}} \dot{x}_{\mathrm{P}}-K_{\delta \mathrm{xp}} x_{\mathrm{P}} \\
\dot{p}_{\mathrm{L}} & =\frac{\beta\left(\alpha+\delta_{0}\right)}{V_{0 \mathrm{~A}} \delta_{0}}\left(q_{\mathrm{L}}-A_{\mathrm{A}} \dot{x}_{\mathrm{P}}-\frac{\delta_{0} K_{\mathrm{Lp} \delta}}{\left(\alpha+\delta_{0}\right)^{2}} p_{\delta}\right. \\
& \left.-\frac{\delta_{0} K_{\mathrm{LpL}}}{\left(\alpha+\delta_{0}\right)^{2}} p_{\mathrm{L}}\right)
\end{aligned}
$$

The choice of $q_{\delta}$ and $q_{\mathrm{L}}$ is such that the nontransformed inputs in terms of shaft velocity and proportional valve flows does not directly influence the pressure level and load pressure gradients, but are contained in $q_{\delta}$ and $q_{\mathrm{L}}$. Control structures using these new inputs can thereby be designed independently of the value and sign of the shaft velocity and of the valve signal allocation.

The input transformation matrix $\mathbf{W}_{1}$ is used to transform $q_{\delta}$ and $q_{\mathrm{L}}$ to the original input signals.

$$
\mathbf{u}=\mathbf{W}_{1} \tilde{\mathbf{u}}
$$

From Eq. (39) and Eq. (40) the inverse input transformation matrix can be obtained as Eq. (44):

$$
\begin{aligned}
\tilde{\mathbf{u}} & =\mathbf{W}_{1}^{-1} \mathbf{u} \\
\tilde{\mathbf{u}} & =\left[\begin{array}{lll}
q_{\mathrm{L}} & q_{\delta} & q_{0}
\end{array}\right]^{T}, \mathbf{u}=\left[\begin{array}{ccc}
\omega_{\mathrm{m}} & q_{\mathrm{vA}} & q_{\mathrm{vB}}
\end{array}\right]^{T} \\
\mathbf{W}_{1}^{-1} & =\left[\begin{array}{ccc}
\frac{\delta_{0} K_{\Lambda \omega}}{\alpha+\delta_{0}} & -\frac{\delta_{0}}{\alpha+\delta_{0}} & \frac{1}{\alpha+\delta_{0}} \\
\left(\alpha+\delta_{0}\right) K_{\Delta \omega} & -\left(\alpha+\delta_{0}\right) & -\frac{\alpha+\delta_{0}}{\alpha} \\
v_{31} & v_{32} & v_{33}
\end{array}\right]
\end{aligned}
$$


The entries $v_{31}, v_{32}, v_{33}$ may be chosen arbitrarily. The flow $q_{0}$ is a flow constraint which is chosen based on the desired distribution of the valve signals. The simplest flow constraint is $q_{0}=0$, which is chosen here.

In Schmidt et al. (2017) main focus is on motion performance, i.e. de-emphasising energy efficiency. The input transformation matrix was constructed such that the valve flows did not influence the load flow in Eq. (40) i.e. ideally the piston motion is only driven by $\omega_{\mathrm{m}}$. This can be obtained by choosing parameters $v_{31}=0$, $v_{32}=1, v_{33}=-1 / \delta_{0}$, and $q_{0}$ according to:

$$
q_{0}=q_{\mathrm{vA}}-\frac{q_{\mathrm{vB}}}{\delta_{0}}=0
$$

The resulting input $\mathbf{u}$ is then given by:

$$
\mathbf{u}^{\mathrm{x}_{\mathrm{P}}}=\mathbf{W}_{1}^{\mathrm{x}_{\mathrm{P}}} \tilde{\mathbf{u}}=\left[\begin{array}{c}
\frac{\alpha+\delta_{0}}{\delta_{0} K_{\Lambda \omega}} q_{\mathrm{L}} \\
\frac{K_{\Delta \omega} \alpha}{\delta_{0} K_{\Lambda \omega}} q_{\mathrm{L}}-\frac{\alpha}{\left(\alpha+\delta_{0}\right)^{2}} q_{\delta} \\
\frac{K_{\Delta \omega} \alpha}{K_{\Lambda \omega}} q_{\mathrm{L}}-\frac{\alpha \delta_{0}}{\left(\alpha+\delta_{0}\right)^{2}} q_{\delta}
\end{array}\right]
$$

The superscript $\mathrm{x}_{\mathrm{P}}$ is added to emphasise that the input transformation is derived to improve motion performance, and is referred to as the original input transformation in the remainder.

In Schmidt et al. (2017) an RGA-analysis of the transformed system, $\tilde{\mathbf{G}}_{\mathrm{C}}$ in Eq. (29) using the transformation matrices $\mathbf{W}_{1}^{\mathrm{x}_{\mathrm{P}}}$ and $\mathbf{W}_{2}$ showed that an almost perfect decoupling in the frequency range below the actuator bandwidths was achieved. A decentralised control strategy of the transformed system is therefore reasonable, where the transformed inputs $q_{\mathrm{L}}$ and $q_{\delta}$ are used to control the transformed inputs $x_{\mathrm{P}}$ and $p_{\delta}$ respectively. These decentralised controllers are designed based on a generic analytical linear controller design approach, presented in Schmidt et al. (2017), capable of calculating appropriate controller parameters regardless of SvSDP drive size. This is done by including physical parameters such as cylinder areas and pump displacements combined with desired relative stability margins in the controller design algorithm. The level pressure ref. generator seen in Fig. 4, is used to generate a $P_{\delta}$ reference. The reference is generated based on which chamber pressure to keep at a reasonable value $p_{\text {set }}=20$ bar and the piston position. In Schmidt et al. (2017) experimental results prove that the SvSDP drive and presented control strategy are capable of maintaining a minimum chamber pressure at $\approx p_{\text {set }}$ while achieving a motion performance at least on the same level as a conventional servo-valve controlled system.

\subsubsection{Energy Efficient Valve Utilisation}

For the presented input transformation matrix, oil is simultaneously throttled through both $2 / 2$ valves, which obviously is not the optimal valve utilisation in terms of energy efficiency. For an energy efficient valve utilisation only oil from the low pressure side should be throttled, which may be achieved by changing the input transformation. It is notable that only the input transformation needs to be changed while the output transformation, controller parameters etc. remain unchanged, as these are used for controlling transformed variables. As such the input transformation is only used to allocate physical inputs from transformed inputs. The input transformation, which only allows oil through the B-side valve can be obtained by defining $v_{31}=0, v_{32}=1, v_{33}=0$ in Eq. (44) and $q_{0}$ as:

$$
q_{0}=q_{\mathrm{vA}}=0
$$

The resulting input $\mathbf{u}^{\mathrm{q}_{\mathrm{vB}}}$ is then given by:

$$
\begin{aligned}
& \mathbf{u}^{\mathrm{q}_{\mathrm{vB}}}=\mathbf{W}_{1}^{\mathrm{q}_{\mathrm{vB}}} \tilde{\mathbf{u}}= \\
& {\left[\begin{array}{c}
\frac{\alpha+\delta_{0}}{\alpha K_{\Delta \omega}+\delta_{0} K_{\Lambda \omega}} q_{\mathrm{L}}+\frac{\alpha}{\left(\alpha+\delta_{0}\right)\left(\alpha K_{\Delta \omega}+\delta_{0} K_{\Lambda \omega}\right)} q_{\delta} \\
\frac{\left(\alpha+\delta_{0}\right) K_{\Delta \omega} \alpha}{\alpha K_{\Delta \omega}+\delta_{0} K_{\Lambda \omega}} q_{\mathrm{L}}-\frac{\delta_{0} K_{\Lambda \omega} \alpha}{\left(\alpha+\delta_{0}\right)\left(\alpha K_{\Delta \omega}+\delta_{0} K_{\Lambda \omega}\right)} q_{\delta}
\end{array}\right]}
\end{aligned}
$$

When only allowing oil through the A-side valve the input transformation can be obtained by defining $v_{31}=$ $0, v_{32}=0, v_{33}=1$ and $q_{0}$ as:

$$
q_{0}=q_{\mathrm{vB}}=0
$$

resulting in the following input transformation:

$$
\begin{aligned}
& \mathbf{u}^{\mathrm{q}_{\mathrm{vA}}}=\mathbf{W}_{1}^{\mathrm{q}_{\mathrm{vA}}} \tilde{\mathbf{u}}= \\
& {\left[\begin{array}{c}
-\frac{\alpha+\delta_{0}}{\left(K_{\Delta \omega}-K_{\Lambda \omega}\right) \delta_{0}} q_{\mathrm{L}}+\frac{1}{\left(\alpha+\delta_{0}\right)\left(K_{\Delta \omega}-K_{\Lambda \omega}\right)} q_{\delta} \\
-\frac{\left(\alpha+\delta_{0}\right) K_{\Delta \omega}}{\left(K_{\Delta \omega}-K_{\Lambda \omega}\right) \delta_{0}} q_{\mathrm{L}}+\frac{K_{\Lambda \omega}}{\left(\alpha+\delta_{0}\right)\left(K_{\Delta \omega}-K_{\Lambda \omega}\right)} q_{\delta} \\
0
\end{array}\right]}
\end{aligned}
$$

Which input transformation Eq. (48) or Eq. (50) to be used depends on which chamber pressure to be controlled to the minimum chamber pressure, $p_{\text {set }}$. This switching condition is defined from the measured load pressure $P_{\mathrm{L}}$ using Eq. (51) setting $P_{\mathrm{A}}=P_{\mathrm{B}}=P_{\text {set }}$

$$
p_{\mathrm{L}_{\mathrm{sw}}}=P_{\text {set }}-\alpha P_{\text {set }}=(1-\alpha) P_{\text {set }}
$$

As illustrated in Fig. 5(a), ideally $P_{\mathrm{A}}=P_{\text {set }}$ for $p_{\mathrm{L}}<$ $p_{\mathrm{L}_{\mathrm{sw}}}$ using the input transformation in Eq. (50) and $P_{\mathrm{B}}=P_{\text {set }}$ for $p_{\mathrm{L}}>p_{\mathrm{L}_{\mathrm{sw}}}$ using the input transformation in Eq. (48). To avoid abrupt jumps in the utilised input transformation method a switching variable $Z$ is defined as:

$$
Z=\left\{\begin{array}{lll}
0 & , & p_{\mathrm{L}}<\left(p_{\mathrm{L}_{\mathrm{sw}}}-Z_{\mathrm{band}}\right) \\
\frac{P_{\mathrm{L}}-p_{\mathrm{L}_{\mathrm{sw}}}+Z_{\mathrm{band}}}{2 Z_{\mathrm{band}}}, & \left(p_{\mathrm{L}_{\mathrm{sw}}}+Z_{\mathrm{band}}\right)>p_{\mathrm{L}}> \\
& & \left(p_{L_{\mathrm{sw}}}-Z_{\mathrm{band}}\right) \\
1 & , & p_{\mathrm{L}}>\left(p_{\mathrm{L}_{\mathrm{sw}}}+Z_{\mathrm{band}}\right)
\end{array}\right.
$$

$\bar{Z}=1-Z ;$ 
In Fig. 5(b) the switching variables are seen as a function of the load pressure. The non-transformed inputs $\mathbf{u}$ are then defined as a weighted sum between $\mathbf{u}^{\mathrm{q}_{\mathrm{vA}}}$ and $\mathbf{u}^{\mathrm{q}_{\mathrm{vB}}}$ according to:

$$
\mathbf{u}=Z \mathbf{u}^{\mathrm{q}_{\mathrm{vB}}}+\bar{Z} \mathbf{u}^{\mathrm{q}_{\mathrm{vA}}}
$$

Eq. (54) is referred to as the energy efficient input transformation in the remainder.

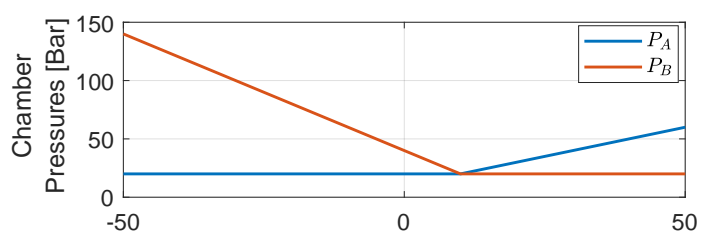

(a) Load Pressure [Bar]

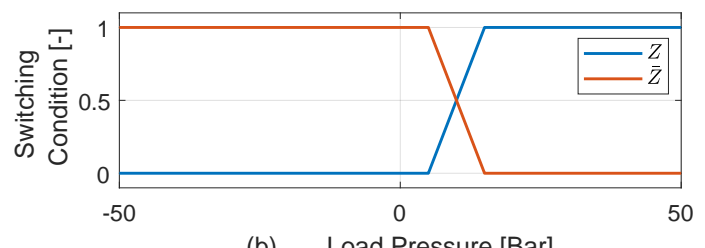

(b) Load Pressure [Bar]

Figure 5: (a) Ideally controlled chamber pressures as a function of load pressure for $p_{\text {set }}=20 \mathrm{bar}$ and $\alpha=0.5$. (b) Input transformation switching variables as a function of load pressure for $Z_{\text {band }}=5$ bar

\subsection{Energy Efficient Pump Sizing}

Assuming that the considered knuckle boom crane should be retrofitted with SvSDP-actuated cylinder drives, it is likely that the hydraulic cylinders remain unchanged thus only replacing the conventional HPU including valves with SvSDP drives. This necessitates that the SvSDP sizing should aim at delivering approximately the same flow amount in the same pressure range as achievable with the conventional Valve Cylinder Drives (VCDs). In Schmidt et al. (2017), external gear pumps have been used as flow suppliers in the SvSDP drive. As these generally operate in a limited pressure range, internal gear pumps are suggested for larger power applications as considered here. The pump sizes selected for the system are of crucial importance in terms of energy efficiency, as these heavily affect both electrical and hydraulic losses. Ten different pump sizes ranging from $16 \mathrm{~cm}^{3} / \mathrm{rev}$ to $125.2 \mathrm{~cm}^{3} / \mathrm{rev}$ (Rexroth, 2010) and (Rexroth, 2013) have been considered for each pump, yielding a total of 1000 different pump size combinations. In this section a selection algorithm aiming on selecting an energy efficient and feasible pump combination is proposed. For doing so, the dominant system losses must be described.

\subsubsection{Dominating Losses}

In Fig. 6 the main losses during operation are shown.

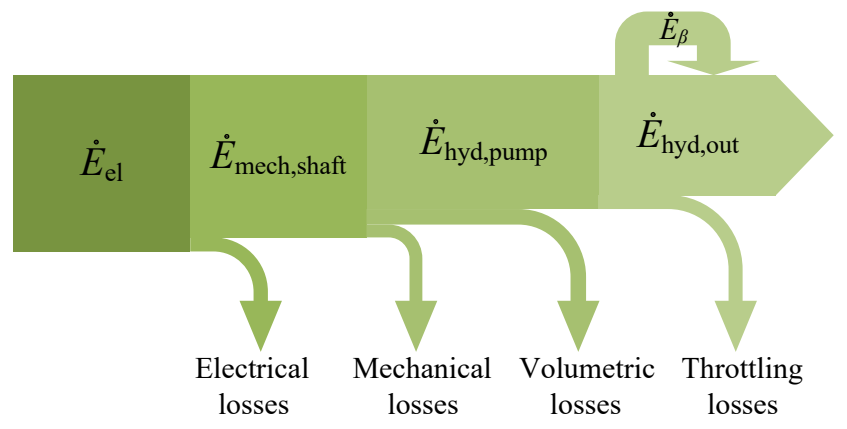

Figure 6: Main losses during operating of the SvSDPactuated cylinder. $\dot{E}_{\beta}$ is power due to the compressibility of the oil.

The electrical losses in the frequency converter and iron losses in the electrical motor are assumed neglectable and thus the only electrical loss included are the Ohmic losses in the electrical motor described by (Willkomm et al., 2014):

$$
\dot{E}_{l, \Omega}=3 R_{\mathrm{cu}} I^{2}=3 R_{\mathrm{cu}}\left(\frac{\tau_{\text {shaft }}}{\tau_{\mathrm{nom}}} I_{\mathrm{nom}}\right)^{2}
$$

$R_{\text {cu }}$ is the winding resistance, and $I_{\text {nom }}$ is the nominal current at the nominal motor torque, $\tau_{\text {nom }}$. These parameters are available from datasheets. $\tau_{\text {shaft }}$ is calculated by:

$$
\tau_{\text {shaft }}= \begin{cases}\left(K_{\mathrm{AQ}}+K_{\mathrm{CQ}}\right) P_{A}-K_{\mathrm{BQ}} P_{\mathrm{B}} & , \quad \omega_{\mathrm{m}} \geq 0 \\ K_{\mathrm{AQ}} P_{\mathrm{A}}-K_{\mathrm{BQ}} P_{\mathrm{B}} & , \quad \omega_{\mathrm{m}}<0\end{cases}
$$

where $K_{\mathrm{AQ}}, K_{\mathrm{BQ}}$ and $K_{\mathrm{CQ}}$ are the theoretical pump displacements $\left[\mathrm{m}^{3} / \mathrm{rad}\right]$.

The mechanical losses due to pump friction have been neglected as no information for the considered internal gear pumps are available. The volumetric losses in the pump are described by:

$$
\dot{E}_{\mathrm{l}, \mathrm{v}}=\left(K_{\mathrm{l}, \mathrm{v} 1} \Delta P+K_{\mathrm{l}, \mathrm{v} 2} \Delta P^{2}\right) \Delta P
$$

where $\Delta P$ is the pressure difference across the pump, and $K_{1, \mathrm{v} 1}$ and $K_{\mathrm{l}, \mathrm{v} 2}$ are leakage parameters. From pump datasheet (Rexroth, 2013), a flow curve for a $16 \mathrm{~cm}^{3} / \mathrm{rev}$ pump at $1450 \mathrm{RPM}$ is available. This has been used to fit the leakage related parameters in Eq. (57), assuming leakage-free flow at $\Delta P=0$. The coherence between the flow curve and the model is seen 
in Fig. 7(a). In Fig. 7(b) corresponding volumetric efficiencies for different speed levels are shown. Identical volumetric efficiencies have been used for all pump sizes considered. This may be a conservative estimation as volumetric efficiencies are assessed to improve for larger pump sizes.
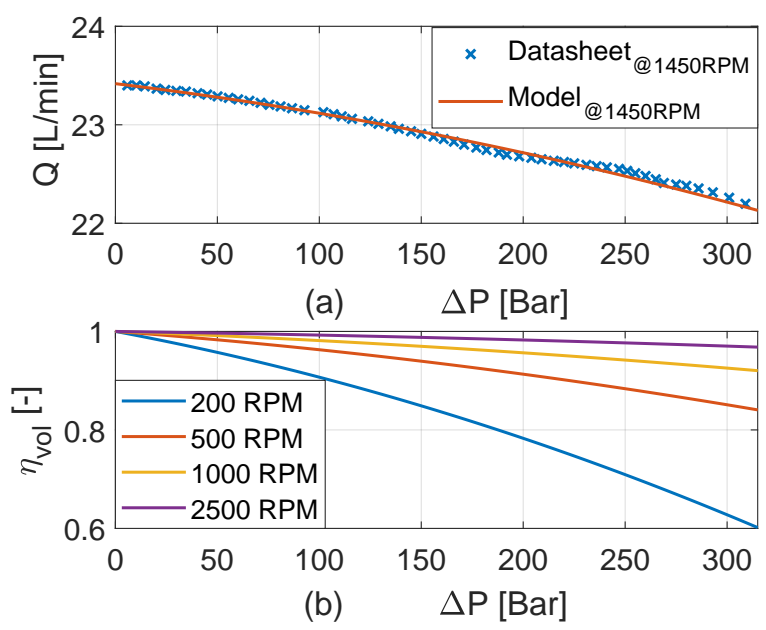

Figure 7: (a) Pump flow model compared to datasheet. (b) Pump volumetric efficiency.

Throttling losses in pipes and hoses as well as over the ideally modelled check valves are neglected. Therefore the throttling losses only involve the oil through the $2 / 2$ proportional valves, described by:

$$
\dot{E}_{\mathrm{l}, \mathrm{th}}=Q_{\mathrm{vA}} \cdot P_{\mathrm{A}}+Q_{\mathrm{vB}} \cdot P_{\mathrm{B}}
$$

\subsubsection{Pump Selection Algorithm}

The Valve Cylinder Drives (VCD) used as a benchmark (see Section 4) for the proposed SvSDP, produces maximum flows of $160 \mathrm{~L} / \mathrm{min}$ to each cylinder. Due to load sensing it is assumed that this can be done independently of the cylinder load pressure. This translates to piston velocities ranging from $-88 \mathrm{~mm} / \mathrm{s}$ to $43 \mathrm{~mm} / \mathrm{s}$ for cylinder 1 and from $-113 \mathrm{~mm} / \mathrm{s}$ to $54 \mathrm{~mm} / \mathrm{s}$ for cylinder 2.

A fundamental difference between a (symmetrical)valve controlled asymmetric cylinder system and the proposed SvSDP system, is that for the SvSDP the pump flows are matched to the cylinder area ratio causing the achievable velocities to be somewhat symmetrically distributed around $0 \mathrm{~mm} / \mathrm{s}$, if utilising the $2 / 2$ valve on the A-side only.

However for energy-efficient valve utilisation it is desired to throttle from the low pressure chamber, which may be any chamber, affecting the achievable piston velocities. E.g. if always throttling from the B-side, the steady state forward velocity is determined by the combined flow of pump $\mathrm{C}$ and $\mathrm{A}$, whereas the retracting velocity is determined by the flow of pump A only as the C-pump is idling. This causes the maximum velocities to be asymmetrical around $0 \mathrm{~mm} / \mathrm{s}$. For the given example the forward velocity is larger than the retracting velocity, exactly opposite of a VCD.

These considerations show that a SvSDP drive sized to achieve VCD comparable retracting velocities, may be heavily oversized in the forward direction, due to idling of the $\mathrm{C}$ pump. The flow requirements (FR) are therefore relaxed in the retracting direction and formulated as:

$$
\begin{aligned}
& \mathrm{FR}^{\omega_{\mathrm{m}} \geq 0}= \begin{cases}1, & \left(\left(Q_{\mathrm{A}}+Q_{\mathrm{C}}\right) \geq 160 \frac{\mathrm{L}}{\min }\right) \\
0 & , \quad \text { otherwise }\left(Q_{\mathrm{B}} / \alpha \geq 160 \frac{\mathrm{L}}{\min }\right)\end{cases} \\
& \mathrm{FR}^{\omega_{\mathrm{m}}<0}= \begin{cases}1, & \left(-Q_{\mathrm{A}} \geq 0.9 \cdot 160 \frac{\mathrm{L}}{\min }\right) \\
0, & \text { and }\left(-Q_{\mathrm{B}} / \alpha \geq 160 \frac{\mathrm{L}}{\min }\right)\end{cases}
\end{aligned}
$$

where $Q_{\mathrm{A}}, Q_{\mathrm{B}}, Q_{\mathrm{C}}$ are pump flows evaluated at maximum allowed pressure and maximum positive/negative pump speed for $\mathrm{FR}^{\omega_{m} \geq 0}$ and $\mathrm{FR}^{\omega_{\mathrm{m}}<0}$ respectively. For a combination of pumps to be feasible $\mathrm{FR}^{\omega_{\mathrm{m}} \geq 0}=$ 1 and $\mathrm{FR}^{\omega_{\mathrm{m}}<0}=1$ is required along with a matchratio $(\chi)$ larger than 1 evaluated at $\pm 500 \mathrm{RPM}$ and 225 bar. For $\chi>1$ a surplus of flow into the cylinder is present. $\chi$ is defined as:

$$
\chi=\left\{\begin{array}{lll}
\frac{Q_{\mathrm{A}}+Q_{\mathrm{C}}}{Q_{\mathrm{B}}} \alpha & , \quad \omega_{\mathrm{m}} \geq 0 \\
\frac{Q_{\mathrm{B}}}{Q_{\mathrm{A}} \alpha} & , \quad \omega_{\mathrm{m}}<0
\end{array}\right.
$$

Evaluating Eq. (59), (60) and Eq. (61) gives a number of feasible designs. Assuming oil always to be throttled from the low pressure side and by neglecting pump leakage the static mismatch flow for each feasible combination can be calculated and throttling losses evaluated using Eq. (58), as a function of shaft speed and $p_{\text {set }}$. Assuming a constant minimum chamber pressure, the shaft torque may be calculated and the Ohmic losses may be evaluated using Eq. (55) as a function of load pressure. By sweeping over the entire pump velocity and load pressure range, the pump combination with the smallest average loss is chosen. The pump selection algorithm is summarised in Fig. 8.

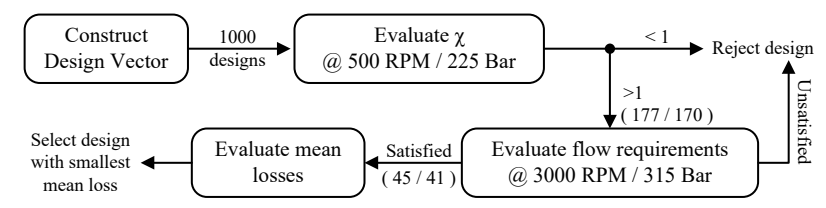

Figure 8: Pump selection algorithm. In parenthesis are shown the number of feasible designs for $\mathrm{SvSDP}_{1}$ and $\mathrm{SvSDP}_{2}$ respectively. 
As the two cylinders have almost equivalent area ratios and the same flow requirement is imposed the selected pump combination is the same for $\operatorname{SvSDP}_{1}$ and $\mathrm{SvSDP}_{2}$. Pump A is selected as having a displacement of $50.7 \mathrm{~cm}^{3} / \mathrm{rev}$ and Pump B and $\mathrm{C}$ are both having a displacement of $32.7 \mathrm{~cm}^{3} / \mathrm{rev}$. The resulting match ratio $\chi$ for $\mathrm{SvSDP}_{1}$ is seen in Fig $9(\mathrm{a})$.
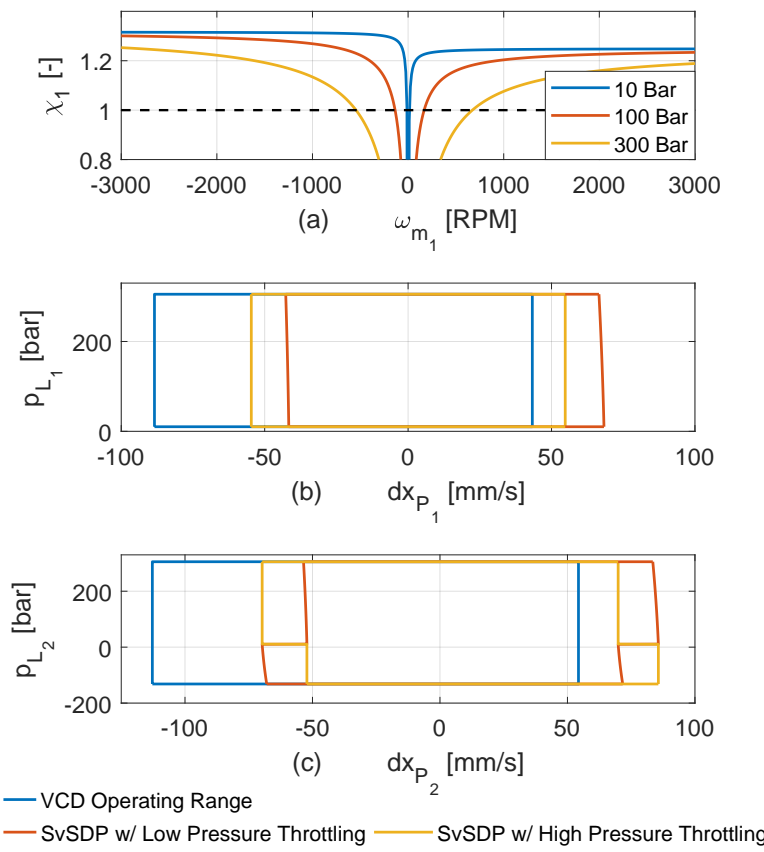

Figure 9: (a) Match ratio for the selected pump combination. (b) Operating range for $\mathrm{SvSDP}_{1}$ compared to VCD. (c) Operating range for $\mathrm{SvSDP}_{2}$ compared to VCD.

The obtained operating ranges, assuming that a suitable electrical motor which does not saturate at corner power requirements, is seen in Fig. 9(b) and (c). The operating ranges are evaluated by assuming that the control structure can maintain a minimum chamber pressure of 20 bar during motion, and using a maximum operating pressure of 315 bar for the internal gear pumps.

Noting that due to the crane structure chamber A will always be load carrying for $\operatorname{SvSDP}_{1}$ while the $\mathrm{SvSDP}_{2}$ should carry the load in both directions, this translates to load forces ranging from 10 to 305 bar $(62$ $\mathrm{kN}$ to $1.88 \mathrm{MN})$ for $\mathrm{SvSDP}_{1}$ and -132 to 305 bar (-0.65 $\mathrm{MN}$ to $1.5 \mathrm{MN}$ ) for $\mathrm{SvSDP}_{2}$ respectively.

Note, that in Fig. 9 operating ranges obtainable if throttling from the high pressure chamber are also depicted. Doing so, alters the achievable operating range, at the cost of larger throttling losses.

\section{Benchmark System}

As mentioned, the benchmarks for the two SvSDP drives are Valve Cylinder Drives (VCDs), conventionally used for actuation of a knuckle boom crane. The hydraulic system structure is shown in Fig. 10, and has also been used in Donkov et al. (2018). The motion of the cylinders is controlled by the directional proportional valves. The two directional valves are pressure compensated and produce maximum flows of 160 L/min. Furthermore, the supply pump has load sensing capabilities. The larger pressure in an inlet chamber selects the outlet pressure setting for the pump $\left(P_{s}=P_{\max }+35\right.$ bar $)$. The counterbalance valves $(\mathrm{CBV})$ are used to prevent the load from overrunning.

For details on the modeling of the benchmark system, component sizes etc., see Donkov et al. (2018).

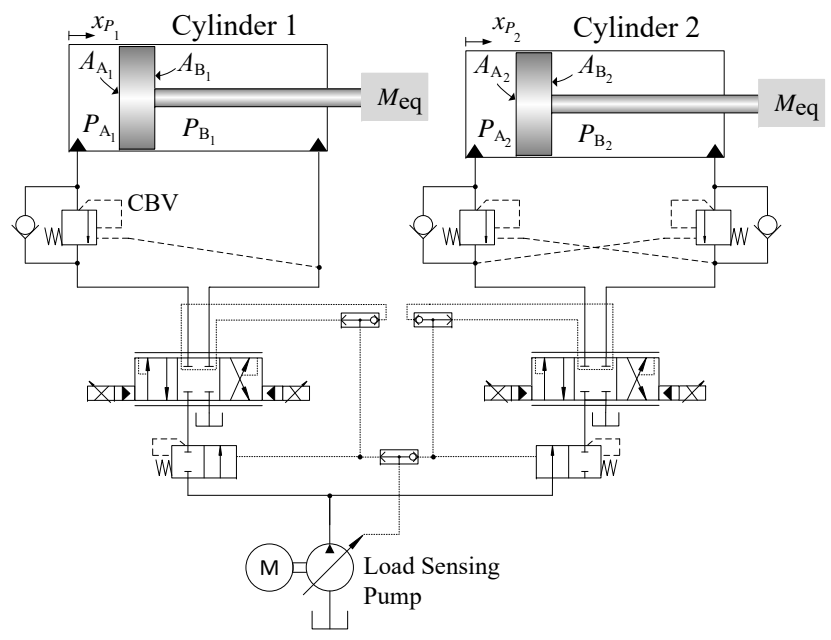

Figure 10: Knuckle Boom Crane hydraulic circuit from Donkov et al. (2018).

\section{Simulation Results}

To compare the performance of the SvSDP concept with the conventional system a simulation study has been conducted. A test case trajectory has been selected where a $5000 \mathrm{~kg}$ payload begins and ends in the same place. In tool center space this can be seen in Fig. 11 taken from Donkov et al. (2018). The trajectory consists of starting the load at point 1 , moving it to point 2 and returning it to point 1 .

In actuator space the trajectory is shown in Fig. 12. Key parameters, such as component sizes, crane dimensions and masses used for the simulation study are found in the Key Parameter List on page 88. 


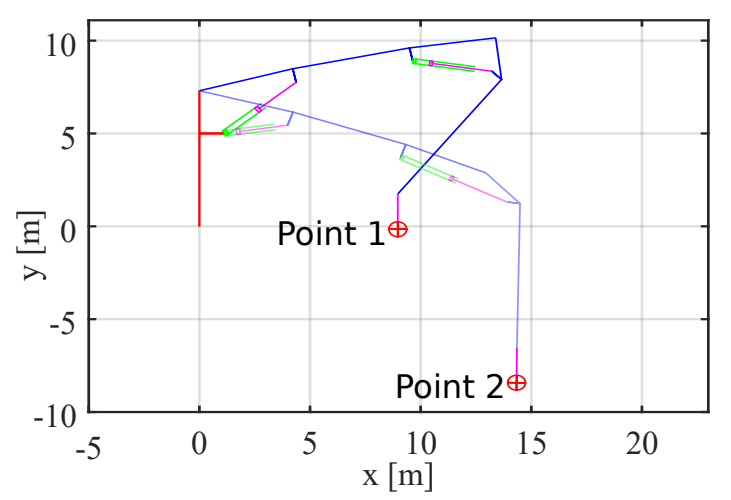

Figure 11: The load is moved from 1 to 2 and back to 1. Donkov et al. (2018).

\subsection{Motion- and Control Stucture Performance}

In Fig. 13 the performance results for the VCDs are shown. It is found that satisfactory motion tracking is achieved, as the maximum position error for cylinder 1 is $9 \mathrm{~mm}(0.4 \%$ of full stroke $)$ and $18 \mathrm{~mm}(0.6 \%$ of full stroke) for cylinder 2. Note, in Fig. 13(e) the valves are almost fully open in the forward direction, meaning that the trajectory is defined close to the forward velocity limits. In Fig. 13(f) the supply pressure $\left(p_{s}\right)$ of the common pump is adjusted accordingly to the valve positions. This explains why during standstill $p_{s}$ oscillates, as the valve positions oscillate around the

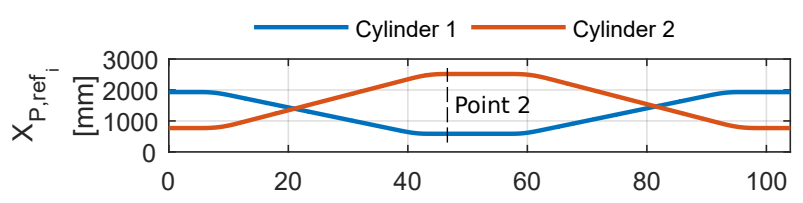

(a) Time $[\mathrm{s}]$

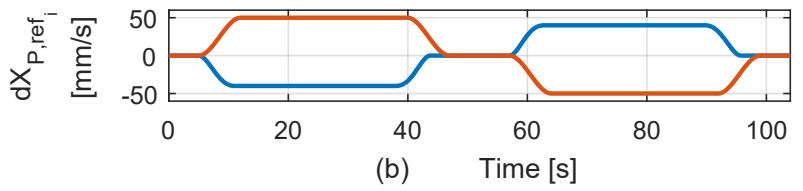

Figure 12: Test case trajectory in actuator space. The start and end point correspond to point 1 in Fig. 11. Point 2 is marked.

0-position.

In Fig. 14 the performance results for the SvSDP drives are shown, using the energy efficient input transformation, Eq. (54). It is seen that motion tracking is on the same level as for the VCDs. The SvSDP performs slightly better for cylinder 1 compared to the VCD system, with a maximum position error of $3 \mathrm{~mm}(0.1 \%$ of full stroke). For cylinder 2 the maximum position error is comparable to the VCD, but during forward motion a slightly larger tracking error is present for the SvSDP. In total it is assessed that the motion performance are on the same level.

From Fig. 14(b) and (d) it is seen that the control structure manages to keep the minimum chamber pres-
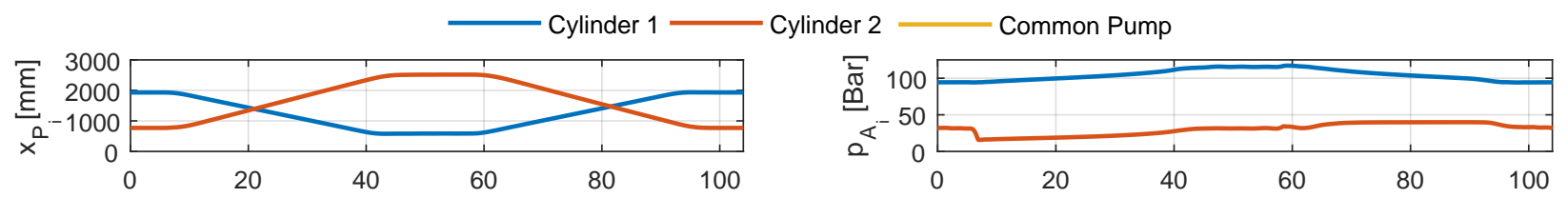

(a) Time [s]

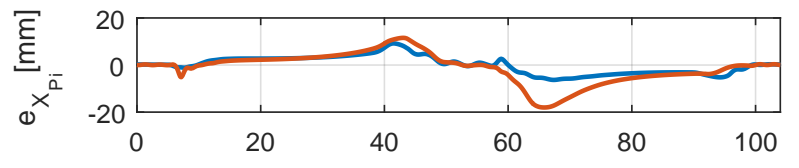

(c) Time $[\mathrm{s}]$

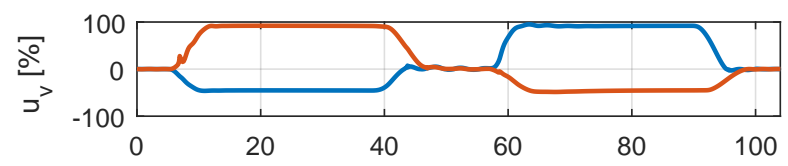

(b) Time $[\mathrm{s}]$

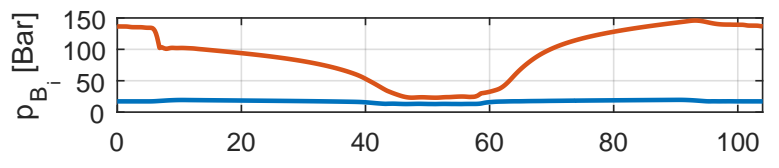

(d) Time $[\mathrm{s}]$

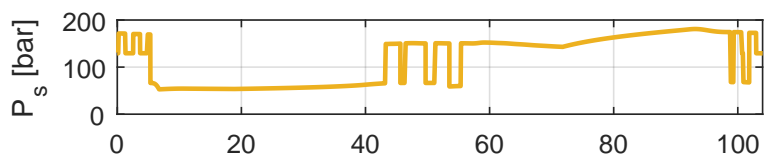

(f) Time $[\mathrm{s}]$
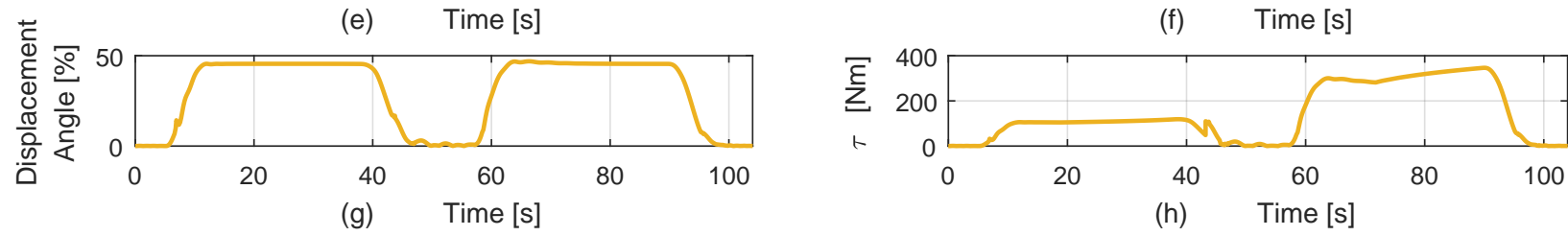

Figure 13: Performance results for the Valve Cylinder Drives (VCDs). 


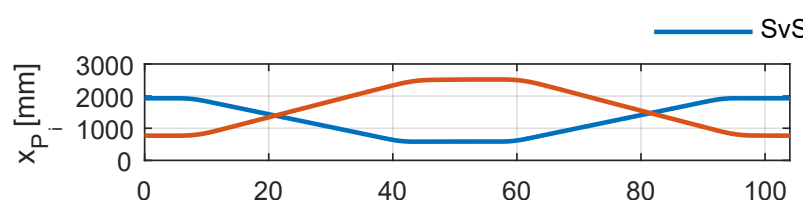

(a) Time [s]

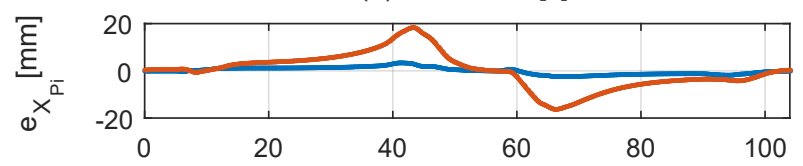

(c) Time $[\mathrm{s}]$

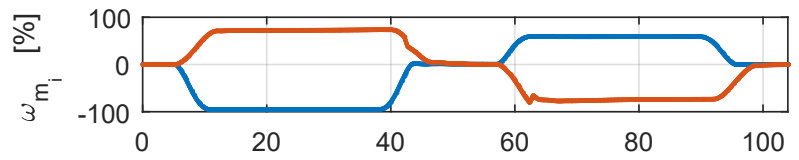

(e) Time $[\mathrm{s}]$

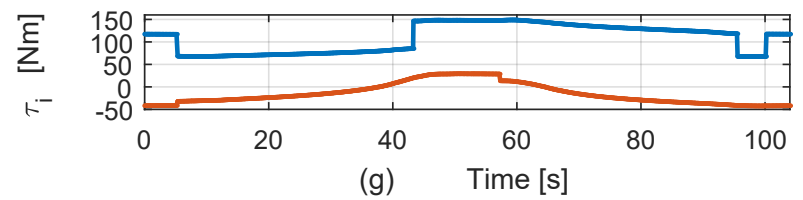

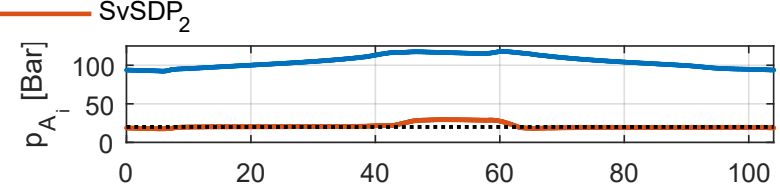

(b) Time $[\mathrm{s}]$

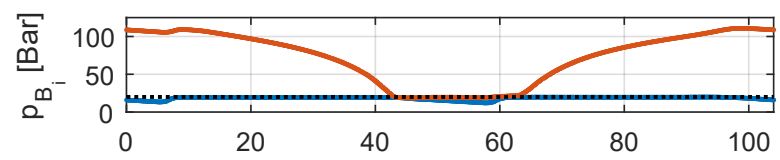

(d) Time $[\mathrm{s}]$

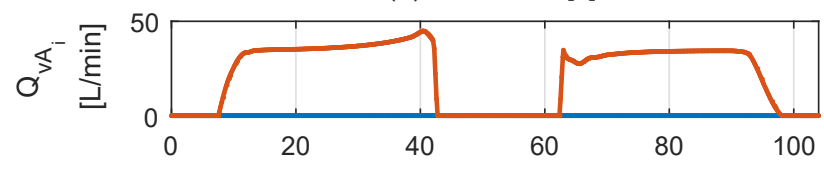

(f) Time [s]

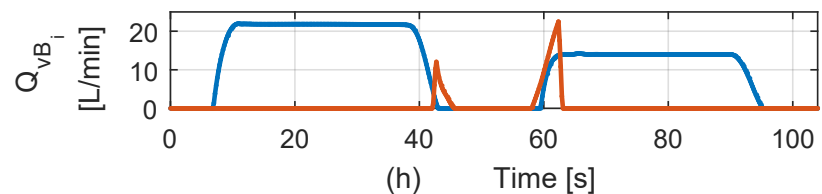

Figure 14: Performance results for SvSDP drives using the energy efficient input transformation in Eq 54 . In (b) and (d) the dotted black line indicates the pressure setting, $p_{\text {set }}$.

sure in each cylinder close to the pressure setting at $p_{\text {set }}=20$ bar. Due to the short duration of standstill at point 1 and 2 in the trajectory and the small leakage flows in the internal gear pumps, even at standstill the minimum chamber pressure is remaining close to the set pressure. In Fig. 14(e) it should be noted, that the SvSDP drives are close to the motor speed saturation limits in the retracting direction as opposed to the VCD. This is due to different obtainable operating ranges, visualised in Fig. 9.

As desired with the energy efficient input transformation, oil is only throttled from the low pressure chamber, see Fig. 14(f) and (h). For $\mathrm{SvSDP}_{1}$ this means that only valve $\mathrm{B}$ is active, as chamber $\mathrm{A}$ is always the load carrying chamber. For $\mathrm{SvSDP}_{2}$ it is seen that chamber B is mainly the load carrying chamber, why oil is throttled from chamber A. However between 40 to 60 seconds the load carrying chamber is interchanged. In this switching process, oil is throttled through both valve A and B as desired. Simulations have also been performed using the original input transformation (Eq.(46)) for the SvSDP drive. Motion tracking and pressure control performance are almost identical to what have been achieved using the energy efficient input transformation (Fig. 14(a)-(d)). The valve flows are however, fundamentally different. The valve flows using the original input transformation are seen in Fig. 15. As desired with the original input transformation oil is throttled using both valves, why a

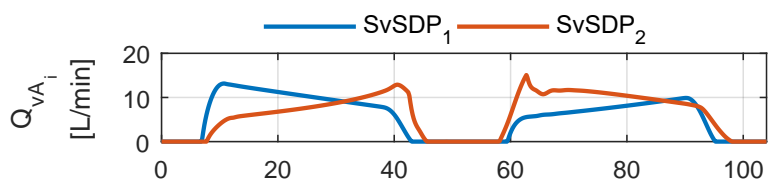

(b) Time $[\mathrm{s}]$

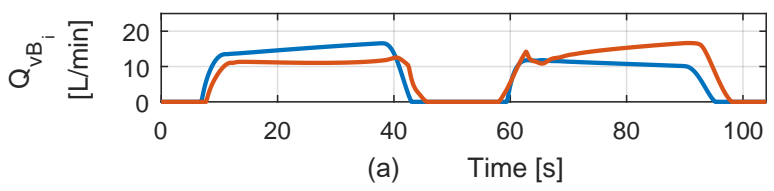

Figure 15: Valve flows for SvSDP drives using original input transformation (Eq (46)). Compare to Fig. 14(f) and (h).

degraded energy efficiency for the original input transformation is to be expected.

Interestingly, the simulation study does not show that the motion tracking performance for the proposed energy efficient input transformation is degraded compared to the original input transformation.

\subsection{Energy Comparison}

Having established that the motion tracking performance is on the same level for the SvSDP and the VCD, it is interesting to compare the energy consumption of the two. Included is also a comparison of the SvSDP drives using the original input and the energy efficient 


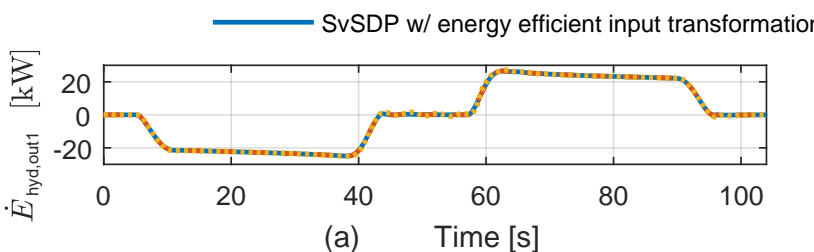

(a) Time $[\mathrm{s}]$

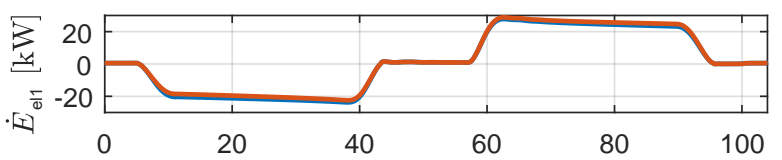

(c) Time $[\mathrm{s}]$
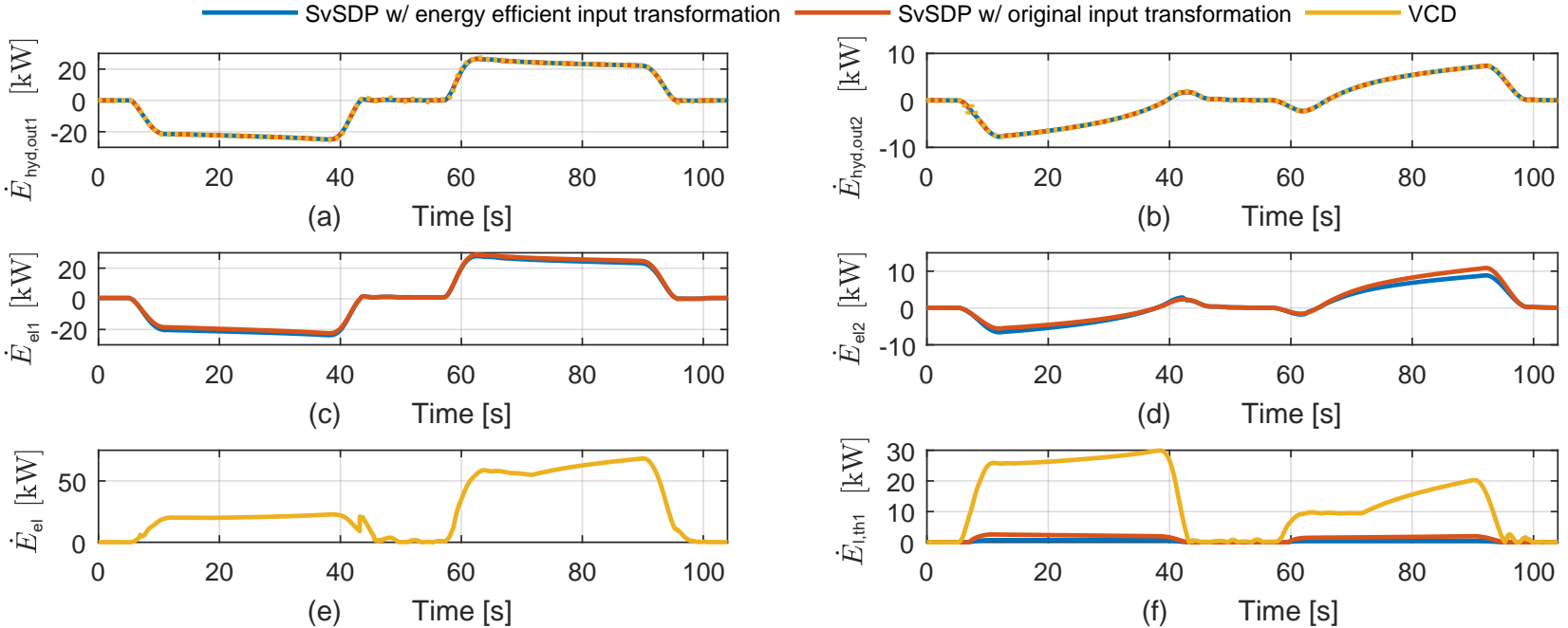

Figure 16: (a) Hydraulic output power cylinder 1. (b) Hydraulic output power cylinder 1. (c) Electrical input power SvSDP 1 . (d) Electrical input power $\mathrm{SvSDP}_{2}$. (e) Electrical input power VCD, common motor. (f) Throttling losses for cylinder 1.

input transformation.

From Fig. 16(a) and (b) it may be seen that the hydraulic output power $\left(F_{\text {hyd }} \cdot \dot{x}_{\mathrm{P}}\right)$ is close to identical for the SvSDP drives and the VCD, which is a necessity for a fair evaluation of energy consumption.

For the VCD the electrical input power cannot be separated between the two cylinders due to the common pump, as opposed to the SvSDP.

Fig. 16(c) and (d) show that the electrical input power for the SvSDP using the original input transformation is slightly higher than using the energy efficient transformation. Note, that from 10 to 40 seconds, the electrical input power is negative meaning the electrical motor effectively functions as a generator, and that a potential for energy recovery is present. As seen in Fig. 16(e), the electrical input power is always positive for the VCD, meaning that even though hydraulic power is available for recovery, the electrical motor still needs to supply power for the system to operate.

Generally, it is seen that electrical input power to the VCD is larger than for the SvSDP even though Fig. 16(c) and (d) should be added to obtain the combined input power for operation of the knuckle boom crane. This difference is primarily due to much larger valve throttling losses, as shown in Fig. 16(f).

Note, that from 10 to 40 seconds the throttling losses for the VCD exceed the input power, because the hydraulic power is negative.

\subsubsection{Energy Efficiency}

For the SvSDP drives it is possible to define the efficiency based on transferred power individually on a drive level as:

$$
\begin{aligned}
& \eta_{i}=\frac{\dot{E}_{\mathrm{Out}_{i}}}{\dot{E}_{\mathrm{in}_{i}}} \quad \dot{E}_{\mathrm{out}_{i}}=\left\{\begin{array}{ccc}
\dot{E}_{\mathrm{hyd}_{\text {out }}} & , & \dot{E}_{\mathrm{el}_{i}} \geq 0 \\
\dot{E}_{\mathrm{el}_{i}} & , & E_{\mathrm{el}_{i}}<0
\end{array}\right. \\
& \dot{E}_{\mathrm{in}_{i}}=\left\{\begin{array}{ccc}
\dot{E}_{\mathrm{el}_{i}} & , \quad \dot{E}_{\mathrm{el}_{i}} \geq 0 \\
\dot{E}_{{\mathrm{hyd}, \mathrm{out}_{i}}}, & \dot{E}_{\mathrm{el}_{i}}<0
\end{array}\right.
\end{aligned}
$$

Utilising Eq. (62) it is possible to calculate the efficiency both when electrical energy is supplied or regenerated during the trajectory for $\operatorname{SvSDP}_{1}$ and $\mathrm{SvSDP}_{2}$ individually.

For the VCD system the energy efficiency is only evaluated when both hydraulic power outputs are positive:

$\eta_{\mathrm{vCD}}=\frac{\dot{E}_{\mathrm{out}, \mathrm{VCD}}}{\dot{E}_{\mathrm{in}, \mathrm{VCD}}}$

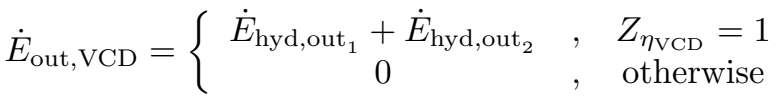

$\dot{E}_{\mathrm{in}, \mathrm{VCD}}=\left\{\begin{array}{ccc}\dot{E}_{\mathrm{el}, \mathrm{VCD}} & , & Z_{\eta_{\mathrm{VCD}}}=1 \\ 0 & , & \text { otherwise }\end{array}\right.$

$Z_{\eta_{\mathrm{VCD}}}=\left\{\begin{array}{ccc}1 & , & \dot{E}_{\mathrm{hyd} \text { out }_{1}}>0 \text { and } \dot{E}_{{\mathrm{hyd}, \text { out }_{2}}}>0 \\ 0, & \text { otherwise }\end{array}\right.$

The evaluated energy efficiencies during the trajectory are visualised in Fig. 17.

It is found that generally the energy efficiency is higher for the SvSDP drives using the energy efficient input transformation compared to the original. Also, both input transformation strategies outperform the VCD. The overall efficiency during the trajectory is 


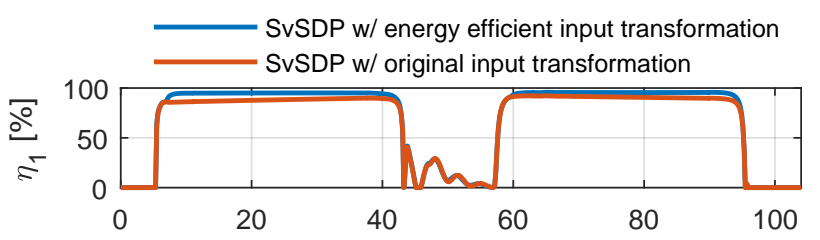

(a) Time [s]

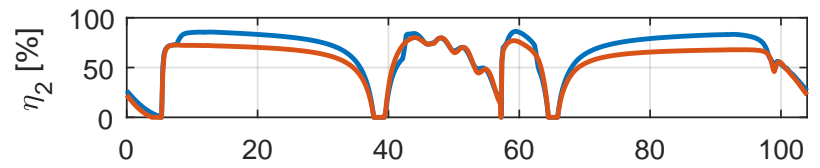

(b) Time [s]

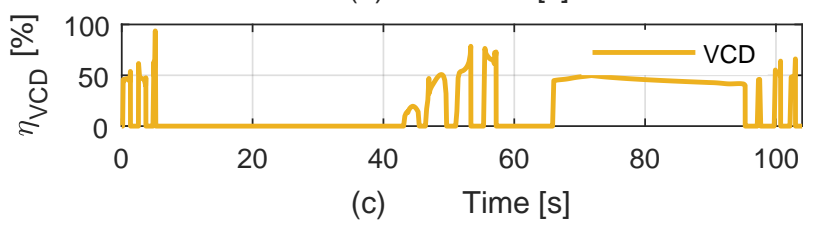

Figure 17: Evaluated energy efficiencies during the trajectory evaluated using Eq. (62) and Eq. (63).

calculated by a time integration of the power expressions from Eq. (62) and Eq. (63), and calculated using $E_{\text {out }} / E_{\text {in }}$ : The result of this is summarised in Tab. 1.

\begin{tabular}{lcc}
\multicolumn{3}{c}{ Energy Efficiency $(\eta)$} \\
\hline SvSDP & \multicolumn{2}{c}{ Input Transformation } \\
& Energy Efficient & Original \\
\cline { 2 - 3 } $\mathrm{SvSDP}_{1}$ & $93.7 \%$ & $88.6 \%$ \\
$\mathrm{SvSDP}_{2}$ & $76.8 \%$ & $65.0 \%$ \\
\hline $\mathrm{VCD}$ & \multicolumn{3}{c}{$44.9 \%$} \\
\hline
\end{tabular}

Table 1: Evaluated energy efficiencies for the test trajectory.

The efficiency of the VCD is significantly smaller compared to the SvSDP drives. As indicated in Fig. 16(f) increased throttling losses are the main reason.

The efficiency of the SvSDP drives using the energy efficient input transformation is larger compared to the original transformation. Also, $\mathrm{SvSDP}_{1}$ is seen to be significantly more efficient than the $\mathrm{SvSDP}_{2}$, for both input transformations. The input energy distributions are visualised in the Sankey diagrams in Fig. 18.

From Fig. 18 it is evident that the degraded energy efficiency for $\mathrm{SvSDP}_{2}$ compared to $\mathrm{SvSDP}_{1}$, is due to increased relative throttling losses. This combined with a smaller amount of converted energy for $\mathrm{SvSDP}_{2}$ explains the smaller efficiency.

Increased throttling losses, due to flow being throttled from both the high and low pressure chamber using the original input transformation, explains the smaller

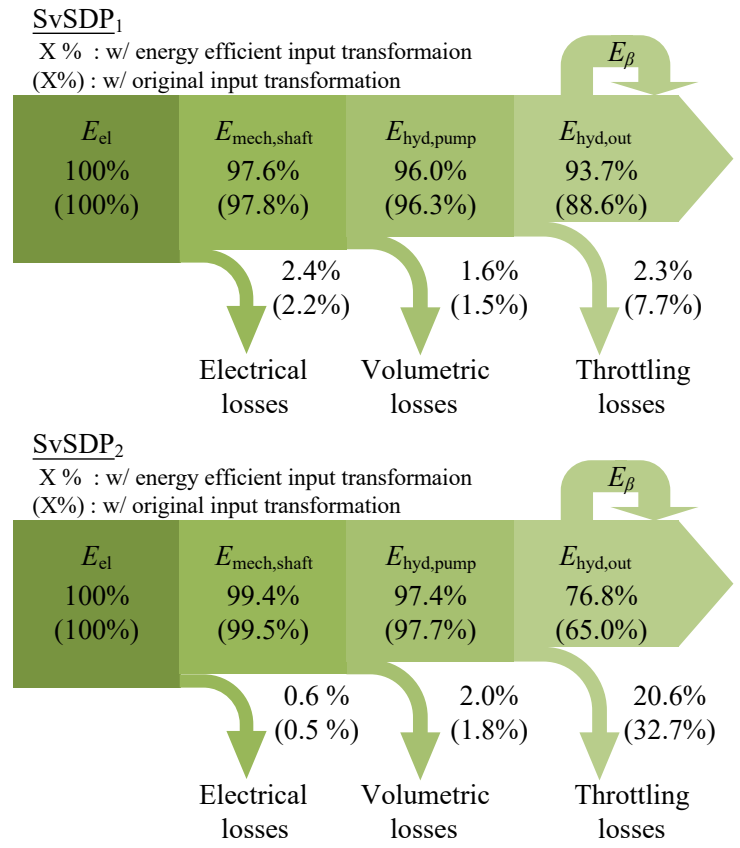

Figure 18: Sankey diagram showing the energy distribution from electrical input to hydraulic output for the SvSDP drives. In parenthesis are the results using the original input transformation.

efficiency compared to the proposed energy efficient input transformation.

\subsubsection{Supplied Electrical Energy}

As seen from Fig. 16 the SvSDP drives are capable of recovering energy as opposed to the VCDs. It is therefore interesting to investigate how much electrical input energy is needed in order for the knuckle boom crane to perform the defined test case trajectory, when assuming ideal energy recovery and storing in the grid. Also, as the efficiencies for the VCD and the SvSDP drives are not defined equivalently, it may be more informative to evaluate and compare the needed input energy instead of efficiency. For the SvSDP system the needed input energy is the sum of the input energy to both $\operatorname{SvSDP}_{1}$ and $\mathrm{SvSDP}_{2}$.

The required input energy during a trajectory is shown in Fig. 19 for the VCD and the SvSDP drives using the energy efficient input transformation.

Tab. 2 summarises the findings from Fig. 19. Clearly a large reduction in required input energy has been achieved for the SvSDP drives compared to the VCD. Even without the possibility of recovering and storing energy, the needed input energy is reduced by $62 \%$, from $0.79 \mathrm{kWh}$ to $0.30 \mathrm{kWh}$. Assuming ideal 


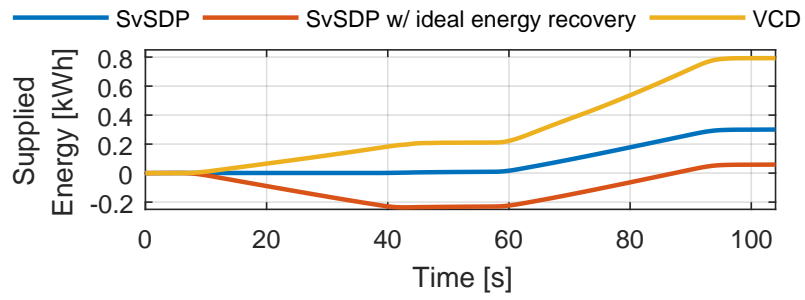

Figure 19: Supplied input energy during a trajectory. The shown SvSDP curves are evaluated using the energy efficient input transformation.

energy recovery the required energy is further reduced to $0.06 \mathrm{kWh}$. This is a reduction of $92 \%$ compared to the VCD.

\begin{tabular}{lll}
\multicolumn{3}{c}{ Supplied Electrical Energy } \\
\hline SvSDP & \multicolumn{2}{c}{ Input Transformation } \\
& Energy Efficient & Original \\
\cline { 2 - 3 } No recovery & $0.30 \mathrm{kWh}$ & $0.35 \mathrm{kWh}$ \\
w/ ideal recovery & $0.06 \mathrm{kWh}$ & $0.10 \mathrm{kWh}$ \\
\hline VCD & \multicolumn{2}{c}{$0.79 \mathrm{kWh}$} \\
\hline
\end{tabular}

Table 2: Supplied input energy for the investigated knuckle boom crane to perform the test trajectory.

Also, the SvSDP drives using the proposed energy efficient input transformation is consuming less input energy compared to the original input transformation. Here a reduction of $14 \%$ is seen if energy cannot be recovered and $40 \%$ if ideal energy recovery is assumed.

This emphasises the importance of proper control considerations. With exactly the same SvSDP system from a hardware point of view, that performs the same motion trajectory with equivalent tracking performance, the energy consumption can be drastically decreased, by proper control efforts.

\section{Discussion}

For the SvSDP drives to be valid alternatives to conventional valve controlled drives, the safety function of the counterbalance valves must be imitated. For the SvSDP drives the risk of over-running loads are reduced, as the pump leakage path is significantly more restrictive than the return path of a proportional valve. However, in emergency situations where input power is lost, a load may drop rapidly as the electrical motor is passively accelerated. For safety critical applications this cannot be tolerated. It is not feasible to utilise counterbalance valves, as these prevent the opportunity of energy recovery. Instead the safety functionality may be achieved by inserting normally closed on/off valves in the main transmission lines.

When evaluating energy efficiencies for the SvSDP drive only three sources of losses have been included. It is therefore unlikely that the stated efficiencies can be achieved in a real world application. However, as only two sources of losses have been included for the Valve Cylinder Drives (VCD) it is expected that the relative difference between the SvSDP and the VCD is representative.

\section{Conclusion}

An application study, investigating the energy saving potential for knucke boom crane actuation has been conducted. Based on the Speed-variable Switched Differential Pump (SvSDP)-concept, which previously has been experimentally verified for low power applications, a system has been designed based on available pump sizes. The control system for the over-actuated SvSDP drive has been modified compared to previous studies, aiming on improving the energy efficiency of the SvSDP drive.

A test case knuckle boom crane trajectory has been defined to evaluate the performance of the SvSDP drives. Simulation results show that motion tracking performance is on a similar level compared to a Valve Cylinder Drive (VCD), while the energy consumption has drastically decreased. This is mainly due to decreased throttling losses, and the opportunity to recover energy in motoring quadrants for the SvSDP. For the given trajectory the VCD consumes $0.79 \mathrm{kWh}$ of electrical energy, while the SvSDP drives consume 62 $\%$ less energy $(0.30 \mathrm{kWh})$ if energy cannot be recovered and $0.06 \mathrm{kWh}(92.4 \%$ decrease $)$ if ideal energy recovery and storage are assumed.

This result suggests that a potential for significant energy savings exists if using the proposed novel pump controlled cylinder drive for knuckle boom crane actuation.

\section{Acknowledgments}

The research in this paper has received funding from The Research Council of Norway, SFI Offshore Mechatronics, project number 237896/O30. 


\section{Key Parameter List}

\begin{tabular}{|c|c|c|}
\hline & Description & Value \\
\hline$L_{\mathrm{CF}}$ & Length, Figure 3 & $13.750 \mathrm{~m}$ \\
\hline$L_{\mathrm{FJ}}$ & Length, Figure 3 & $9.421 \mathrm{~m}$ \\
\hline$x_{\min _{1}}$ & Cylinder Length, Fig. 3 & $2.333 \mathrm{~m}$ \\
\hline$x_{\min _{2}}$ & Cylinder Length, Fig. 3 & $2.846 \mathrm{~m}$ \\
\hline$m_{1}$ & Mass, Fig. 3 & $6000 \mathrm{~kg}$ \\
\hline$m_{2}$ & Mass, Fig. 3 & $3300 \mathrm{~kg}$ \\
\hline$m_{3}$ & Payload, Fig. 3 & $5000 \mathrm{~kg}$ \\
\hline$m_{\text {cyl1 }}$ & Cylinder Mass, Fig. 3 & $2000 \mathrm{~kg}$ \\
\hline$m_{\mathrm{cyl} 2}$ & Cylinder Mass, Fig. 3 & $2000 \mathrm{~kg}$ \\
\hline$D_{\mathrm{A}_{2}}$ & Piston Diameter & $0.28 \mathrm{~m}$ \\
\hline$D_{\mathrm{B}_{2}}$ & Rod Diameter & $0.2 \mathrm{~m}$ \\
\hline$D_{\mathrm{A}_{2}}$ & Piston Diameter & $0.25 \mathrm{~m}$ \\
\hline$D_{\mathrm{B}_{2}}$ & Rod Diameter & $0.18 \mathrm{~m}$ \\
\hline$K_{\mathrm{AQ}}$ & Pump Displacement & $50.7 \frac{\mathrm{cm}^{3}}{\mathrm{rev}_{2}}$ \\
\hline$K_{\mathrm{BQ}}$ & Pump Displacement & $32.7 \frac{\mathrm{cm}^{3}}{\mathrm{rev}}$ \\
\hline$K_{\mathrm{CQ}}$ & Pump Displacement & $32.7 \frac{\mathrm{cm}^{3}}{\mathrm{rev}}$ \\
\hline$R_{\mathrm{cu}}$ & Winding Resistance & $0.06 \Omega$ \\
\hline$\tau_{\text {nom }}$ & Standstill torque & $342 \mathrm{Nm}$ \\
\hline$I_{\text {nom }}$ & Standstill current & $135.5 \mathrm{~A}$ \\
\hline$\omega_{\mathrm{v}_{i}}$ & Bandwidth & $60 \mathrm{~Hz}$ \\
\hline$\omega_{\mathrm{vA}_{i}}$ & Bandwidth & $20 \mathrm{~Hz}$ \\
\hline$\omega_{\mathrm{vB}_{i}}$ & Bandwidth & $20 \mathrm{~Hz}$ \\
\hline$\zeta_{\mathrm{v}_{i}}$ & Damping ratio & 0.5 \\
\hline$\zeta_{\mathrm{vA}_{i}}$ & Damping ratio & 1 \\
\hline$\zeta_{\mathrm{vB}_{i}}$ & Damping ratio & 1 \\
\hline$B_{\mathrm{v}_{i}}$ & Viscous friction coefficient & $40000 \frac{\mathrm{Ns}}{\mathrm{m}}$ \\
\hline$\omega_{\mathrm{m}, \max _{i}}$ & Max. electric motor speed & 3000 RPM \\
\hline$\omega_{\mathrm{m}, \min _{i}}$ & Min. electric motor speed & $-3000 \mathrm{RPM}$ \\
\hline$V_{\mathrm{AO}_{i}}$ & Initial volume & $80 \mathrm{~L}$ \\
\hline$V_{\mathrm{B}_{i}}$ & Initial volume & $80 \mathrm{~L}$ \\
\hline$Z_{\text {band }}$ & Switching range & 1 bar \\
\hline
\end{tabular}

\section{References}

Çaliskan, H., Balkan, T., and Platin, B. E. A Complete Analysis for Pump Controlled Single Rod Actuators. 10th International Fluid Power Conference, 2016. pages 119-132. doi:10.13140/RG.2.2.13163.75046.

Donkov, V., Andersen, T., Pedersen, H., and Ebbesen, M. Application of model predictive control in disrete displacement cylinders to drive a knuckle boom crane. submitted to the 10th Ph.D. Symposium on Fluid Power, 2018.

Hagen, D., Pawlus, W., Ebbesen, M. K., and Andersen, T. O. Feasibility study of electromechanical cylinder drivetrain for offshore mechatronic systems.
Modeling, Identification and Control, 2017. 38(2):59. doi:10.4173/mic.2017.2.2.

Hedegaard Hansen, A., F Asmussen, M., and Bech, M. M. Model predictive control of a wave energy converter with discrete fluid power power take-off system. Energies, 2018. 11(3):635. doi:10.3390/en11030635.

Huova, M., Aalto, A., Linjama, M., Huhtala, K., Lantela, T., and Pietola, M. Digital hydraulic multi-pressure actuator-the concept, simulation study and first experimental results. International Journal of Fluid Power, 2017. 18(3):141-152. doi:10.1080/14399776.2017.1302775.

Järf, A., Minav, T., and Pietola, M. Nonsymmetrical Flow Compensation Using Hydraulic Accumulator. In Proceedings of the 9th FPNI Ph.D. Symposium on Fluid Power FPNI2016. pages 1-6, 2016. doi:10.1115/FPNI2016-1516.

Kogler, H. and Scheidl, R. Energy efficient linear drive axis using a hydraulic switching converter. Journal of Dynamic Systems, Measurement, and Control, 2016. 138(9):091010. doi:10.1115/1.4033412.

Linjama, M. and Huhtala, K. Digital hydraulic power management system-towards lossless hydraulics. In Proceedings of the Third Workshop on Digital Fluid Power. pages 13-14, 2010.

Linjama, M., Vihtanen, H.-P., Sipola, A., and Vilenius, M. Secondary controlled multi-chamber hydraulic cylinder. In The 11th Scandinavian International Conference on Fluid Power, SICFP, volume 9. pages 2-4, 2009 .

Michel, S. and Weber, J. Energy-efficient electrohydraulic compact drives for low power applications. Fluid Power and Motion Control - FPMC 2012, 2012. pages $93-107$.

Pedersen, H. C., Schmidt, L., Andersen, T. O., and Brask, M. H. Investigation of new servo drive concept utilizing two fixed displacement units. JFPS International Journal of Fluid Power System, 2014. 8(1):1-9. doi:10.5739/jfpsij.8.1.

Quan, Z., Quan, L., and Zhang, J. Review of energy efficient direct pump controlled cylinder electro-hydraulic technology. Renewable and Sustainable Energy Reviews, 2014. 35:336-346. doi:10.1016/j.rser.2014.04.036.

Rahmfeld, R. Development and Control of Energy Saving Hydraulic Servo Drives for Mobile Systems. Fortschrittberichte VDI / 12: Verkehrstechnik, 
Fahrzeugtechnik. VDI-Verlag, 2002. PhD. Dissertation.

Rexroth, B. A. RE10227. Internal gear pump, fixed displacement. Type PGH. 2010. URL https://dc-us.resource.bosch.com/media/us/ products_13/product_groups_1/industrial_ hydraulics_5/pdfs_4/re10227.pdf.

Rexroth, B. A. Internal gear pump PGH Fixed displacement Series 2X. 2013. URL https://dc-us.resource.bosch.com/media/us/ products_13/product_groups_1/industrial_ hydraulics_5/pdfs_4/re10223.pdf.

Schmidt, L., Groenkjaer, M., Pedersen, H. C., and Andersen, T. O. Position Control of an Over-Actuated Direct Hydraulic Cylinder Drive. Control Engineering Practice, 2017. 64:1-14. doi:10.1016/J.CONENGPRAC.2017.04.003.
Schmidt, L., Roemer, D. B., Pedersen, H. C., and Andersen, T. O. Speed-variable switched differential pump system for direct operation of hydraulic cylinders. In ASME/BATH 2015 Symposium on Fluid Power and Motion Control. American Society of Mechanical Engineers, 2015. doi:10.1115/FPMC20159575 .

Vukovic, M., Leifeld, R., and Murrenhoff, H. Steam-a hydraulic hybrid architecture for excavators. In 10th International Fluid Power Conference (10. IFK). pages 151-162, 2016.

Willkomm, J., Wahler, M., and Weber, J. Quadratic Programming to Optimize Energy Efficiency of Speed- and Displacement-Variable Pumps. In 8th FPNI Ph.D Symposium on Fluid Power. ASME, 2014. doi:10.1115/FPNI2014-7802. 